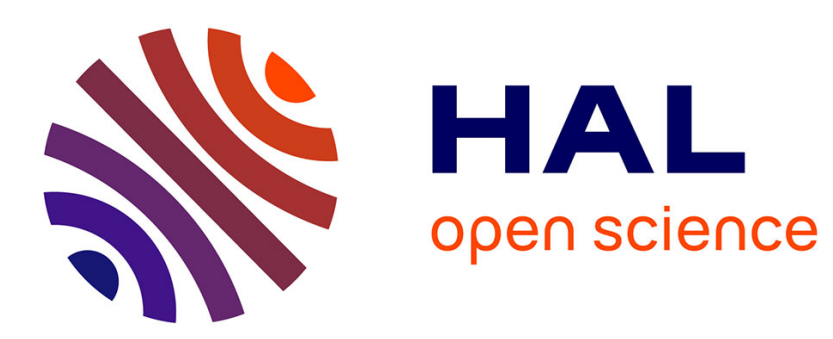

\title{
Diagrammatic Theory of Linear and Non-Linear Optics for Composite Systems
}

T Noblet, Bertrand Busson, C Humbert

\section{To cite this version:}

T Noblet, Bertrand Busson, C Humbert. Diagrammatic Theory of Linear and Non-Linear Optics for Composite Systems. Physical Review A, 2021, 104, pp.063504. 10.1103/PhysRevA.104.063504 . hal-03450820

\section{HAL Id: hal-03450820 \\ https://hal.science/hal-03450820}

Submitted on 26 Nov 2021

HAL is a multi-disciplinary open access archive for the deposit and dissemination of scientific research documents, whether they are published or not. The documents may come from teaching and research institutions in France or abroad, or from public or private research centers.
L'archive ouverte pluridisciplinaire HAL, est destinée au dépôt et à la diffusion de documents scientifiques de niveau recherche, publiés ou non, émanant des établissements d'enseignement et de recherche français ou étrangers, des laboratoires publics ou privés. 


\title{
Diagrammatic Theory of Linear and Non-Linear Optics for Composite Systems
}

\author{
T. Noblet, ${ }^{1,2}$ B. Busson, ${ }^{1}$ and C. Humbert ${ }^{1}$ \\ ${ }^{1}$ Université Paris-Saclay, CNRS, Institut de Chimie Physique, UMR 8000, 91405 Orsay, France. \\ ${ }^{2}$ GRASP-Biophotonics, CESAM, University of Liege, \\ Institute of Physics, Allée du 6 août 17, 4000 Liège, Belgium.
}

(Dated: November 26, 2021)

\begin{abstract}
We present a general formalism to model and calculate linear and nonlinear optical processes in composite systems, based on a graphical representation of light-matter interactions by loop diagrams associated to Feynman rules. Through this formalism, we recover the usual second-order response of a simple system by drawing four times less loop diagrams than doubled-sided ones. For composite systems, we introduce coupling hamiltonians between subsystems (for example a molecule and a substrate), graphically represented by virtual bosons. In this way, we enumerate all the diagrams describing the second-order response of the system and show how to select those relevant for the calculation of the molecular second-order hyperpolarizabilities under the influence of the substrate, including effective second-order contributions from the molecular third-order response. As it applies to all nonlinear processes and an arbitrary number of interacting partners, this representation provides a general frame for the calculation of the nonlinear response of arbitrarily complex systems.
\end{abstract}

Since the emergence of the quantum theory of light, [1] and for several decades now, nonlinear optics has unveiled the existence of couplings within matter [2, 3] thanks to many laser-based techniques of optical characterization, such as second harmonic generation (SHG) [4, 5], sum-frequency generation (SFG), [6-9] coherent and uncoherent Raman scattering [10]. The popularity of these techniques lies in their spectroscopic applications. As experimental tools, infrared-visible SFG and Raman spectroscopies are routinely used in various fields of chemical physics and physical chemistry, largely employed for probing the vibrational structures of molecules [11, 12], and their vibronic structures as for doubly resonant SFG and resonant Raman spectroscopies [13-16]. Although SFG is a powerful technique, it requires ordered and noncentrosymmetric samples. This is why the systems are often voluntarily simplified and reduced to the deposition of a molecular species onto a solid substrate which does not exhibit any optical activity over the probed infrared (IR) and visible ranges. These fall within the family of simple systems made of a single IR/visible-active molecular species, characterized by its own vibrational and electronic structures. As a matter of fact, few publications have so far tackled the less conventional family of composite and hybrid systems, made up of several IR/visible-active species. These differ from simple systems as soon as the local environment (e.g. the substrate) is capable of interacting with the molecules, or reacting to the IR or visible excitations. We may cite for instance substrate/molecule interfaces wherein the substrate may be an insulating [17] or a metallic plane surface [18-25], an electrode [26], or a charged surface [2730]. Further, more complex samples can also be obtained by adding IR/vis-active inorganic species to the system. This includes plasmonic nanostructures [31-36] and semiconductor quantum dots [37-39]. For all these composite systems, the vibrational and electronic responses do not only arise from the molecular species, but are split between the various components (i.e. the subsystems) of the sample. For example, the IR beam may probe the vibrational structure of the molecules while the visible light beam excites the electronic properties of the substrate or the inorganic species. These shared processes essentially differ from doubly resonant SFG for which the molecular species alone experiences vibrational, electronic and vibronic resonances [40-42]. Experimentally, the shared processes become essential when both the IR and visible colours are tunable in a two-colour SFG setup: each sub-process may become resonantly excited or remain nonresonant, at will. The question is then to understand how the complete SFG process is linked to (thus induced, enhanced or modified by) the couplings between the respective vibrational and electronic structures of the subsystems. In this article, we therefore establish the theoretical tools to account for the nonlinear SFG response of any bipartite organic/inorganic system, thus composed of two interacting subunits (Figure 1). This theory may be extended to an arbitrary number of interacting subsystems and to other nonlinear optical processes.

To date, there is no global and generic formalism to analytically compute the nonlinear responses of such bipartite systems. Each team has developed its own methods, so that there are almost as many formalisms as samples [29, 38, 43]. For a bipartite system like a substrate/molecule interface, the second- and third-order hyperpolarizabilities $\beta$ and $\gamma$ of each subsystem are modeled through phenomenological approaches or approximate numerical techniques. With two input frequencies $\omega_{1}$ and $\omega_{2}$, the SFG second-order hyperpolarizability $\beta\left(\omega_{1}, \omega_{2}\right)$ is often simply described as deriving from the sum of two contributions respectively associated to the substrate and the molecules [6, 20, 33, 44]:

$$
\beta=\beta_{\mathrm{sub}}+\beta_{\mathrm{mol}}
$$




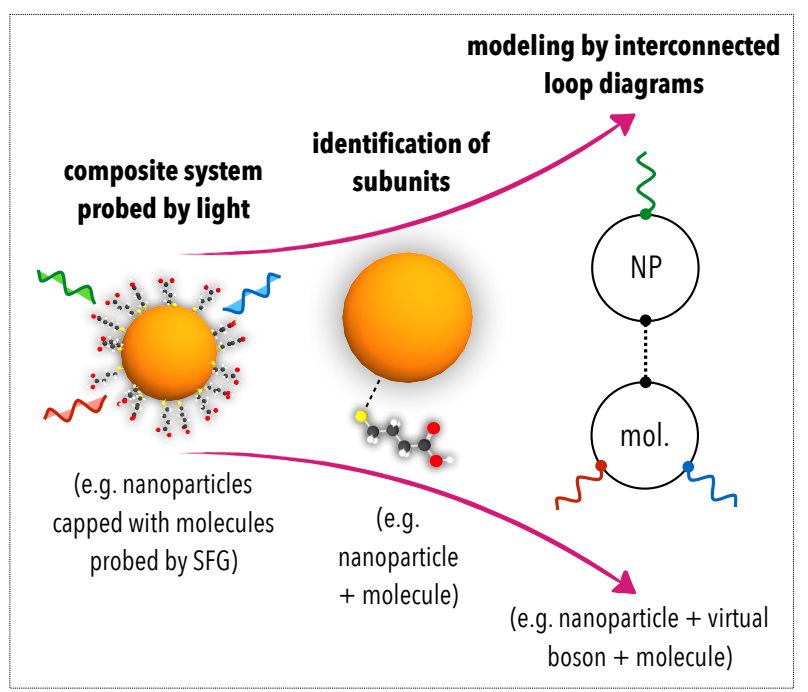

FIG. 1. Diagrammatic modeling of composite systems. The diagrammatic theory is based on our ability to split a composite system into several subunits whose interactions are then embodied by virtual bosons deriving from well-known coupling hamiltonians. Here we illustrate our method with the case of nanoparticles capped by organic molecules and probed by sum-frequency generation (SFG) consisting in the combination of three photons (green, red and blue, in the picture).

First, this equation does not have a firmly established theoretical rationale as both subsystems interact with each other. A third $\beta$ component is sometimes added to account for these interactions [44]. Second, this arbitrary splitting is misleading because the amplitude of the molecular contribution $\beta_{\text {mol }}$ itself depends on the substrate response, as evidenced by previous experimental works in IR-vis SFG spectroscopy [19, 20, 25, 33, 37]. This is why $\beta_{\text {mol }}$, usually modeled by a lorentzian resonance (with respect to the IR frequency $\omega_{2}$ ), is sometimes modulated by an unknown coefficient $A_{v}\left(\omega_{1}, \omega_{2}\right)$ coming from the interactions between the substrate and the molecules [20]:

$$
\beta_{\mathrm{mol}}\left(\omega_{1}, \omega_{2}\right)=\sum_{v} \frac{A_{v}\left(\omega_{1}, \omega_{2}\right)}{\omega_{2}-\omega_{v}+\imath \Gamma_{v}}
$$

where $\omega_{v}$ is the eigenfrequency of the molecular vibration mode $|v\rangle$, and $\Gamma_{v}$ the associated damping constant. In addition, in presence of a static electric field $E_{0}$ arising from an interfacial potential, a third contribution is commonly added to equation (1) [27, 45]:

$$
\begin{aligned}
\beta\left(\omega_{1}, \omega_{2}\right)= & \beta_{\mathrm{sub}}\left(\omega_{1}, \omega_{2}\right)+\beta_{\mathrm{mol}}\left(\omega_{1}, \omega_{2}\right) \\
& +\gamma\left(\omega_{1}, \omega_{2}, 0\right) E_{0},
\end{aligned}
$$

where $\gamma$ is the third-order hyperpolarizability of the interface, which is not explicitly known since it may also depend on the interplay between the substrate and the molecules.
Quantum mechanics provides in principle an effective formalism to derive the analytical expressions of $\beta$ and $\gamma$ through the perturbation expansion of the density matrix of the system [46]. This theoretical approach is operative in the case of simple systems, for example purely molecular. Besides, a diagrammatic formalism has been developed in order to lead the calculations. These graphical depictions, known as the double-sided Feynman diagrams, reproduce the mechanism of perturbation expansion of the density matrix [46-48]. However, this diagrammatic representation of nonlinear optics fails to account for the complex case of the aforementioned composite systems because it is difficult to determine the total density matrix of such a system, that is to deduce the quantum eigenstates of the whole system from those of its subunits. The issue lies in the treatment of the interactions between the subsystems. The problem could be overcome by considering each subunit as described by a double-sided Feynman diagram interacting with the others. Hence, the idea would consist in building a complex diagram, accounting for the composite system, from the double-sided diagrams assigned to each subunit. However, a combination of double-sided diagrams is not a double-sided diagram, so that it is impossible to apply the computation method established for the subunits to the composite system. Given this framework, we have decided to leave aside the double-sided diagrams and go back to the foundations of Feynman diagrams as introduced in solid-state physics in order to treat the manybody problem [49-52]. The loop Feynman diagrams, as introduced in this context, have two great advantages: first, the combination of loop diagrams is a loop diagram and, second, the implementation of interactions between loops only requires the knowledge of the coupling hamiltonians. Henceforth, these loop diagrams enable us to explicitly couple nonlinear optics (i.e. light) with solidstate physics (i.e. matter), accounting for light-matter interactions (between light and a composite system) and matter-matter interactions (between the subunits of this system).

In this paper, we present a global method to analytically compute the exact nonlinear response functions $\beta$ and $\gamma$ for any composite system through the use of loop Feynman diagrams. Especially, we formally show that all the phenomenological contributions $\beta_{\text {sub }}, \beta_{\text {mol }}$ and $\gamma$ can be derived from a single formalism, independently of the natures of the subsystems. Indeed, we give meaning to equation (3) and demonstrate that it is possible to explicitly determine coupling coefficients such as $A_{v}$ (equation (2)). Even though we illustrate the method in the practical case of substrate/molecule bipartite systems probed by IR-vis SFG spectroscopy, by enumerating, drawing and calculating all the Feynman diagrams that it is possible to generate, it can be generalized to other kinds of hybrid systems made of more than two subunits and to other nonlinear processes, e.g. SHG and Raman scatter- 
ing. The method and results presented here naturally rely on the solid state physics formalism and straightforwardly apply to linear and nonlinear processes taking place in objects relevant to this field (i.e. a statistical set of indistinguishable fermions at thermal equilibrium, for example a metal surface), but we show that they equally apply to molecules and atoms after elementary generalization. With this article, our goal is to establish a unified and complete theoretical justification to all the secondorder nonlinear coupling processes within composite systems, and to propose a practical method for everyone to compute the nonlinear response functions of their own composite systems.

\section{FOUNDATIONS OF THE METHOD}

In order to understand the link between nonlinear optics and loop Feynman diagrams, we begin with the definitions of the underlying mathematical tools. Thus, this first section gradually introduces the concepts of optical response functions, Green's functions and Matsubara frequencies. For the sake of brevity and clarity, we get straight to the point by providing only the absolutely necessary theoretical elements. Thereafter, we outline in a synthetic way the general and practical method to build loop diagrams and to compute hyperpolarizabilities for any kind of composite system.

Green's functions in optics and solid-state physics. Within the dipolar approximation, probing matter with light consists in examining how the dipole moment $\mathbf{p}$ reacts to a local exciting electric field $\mathbf{E}$. In frequency space, the linear response theory teaches that there exists a tensor $\boldsymbol{\alpha}=\left(\alpha_{i j}\right)$ so that [46]:

$$
\mathbf{p}(\omega)=\boldsymbol{\alpha}(\omega) \mathbf{E}(\omega)
$$

In time domain, this gives: $\mathbf{p}(t)=\boldsymbol{\alpha} * \mathbf{E}(t)$, where $*$ depicts the convolution product. Further on, the use of intense light allows studying the behaviour of matter beyond the linear dielectric regime. In this case, a nonlinear dipole moment is induced in the material which reveals the existence of second- and third-order response tensors, $\boldsymbol{\beta}=\left(\beta_{i j k}\right)$ and $\boldsymbol{\gamma}=\left(\gamma_{i j k l}\right)$, so that [46]:

$$
\begin{aligned}
\mathbf{p}(t) & =\boldsymbol{\alpha} * \mathbf{E}(t) \\
& +\boldsymbol{\beta} * \mathbf{E} \otimes \mathbf{E}(t)+\boldsymbol{\gamma} * \mathbf{E} \otimes \mathbf{E} \otimes \mathbf{E}(t),
\end{aligned}
$$

where $\otimes$ depicts the tensor product. As a response function, each $\alpha_{i j}, \beta_{i j k}$ and $\gamma_{i j k l}$ is a Green's function and thus belongs to a large family of functions which exceeds the scope of optics [53]. We only remind that a Green's function is defined, for a given linear differential operator $\mathfrak{D}$, as the function $f$ satisfying $\mathfrak{D} f(t)=\delta(t)$, where $\delta$ is the Dirac function. It means that the knowledge of a Green's function is equivalent to that of a differential operator driving the time evolution of a given system in response to an excitation $\delta(t)$. In solid-state physics, Green's functions are used to describe the propagation of quantum states after excitation of the system [49-52]. More precisely, for a quantum system characterized by the states $|n\rangle, n \in \mathbb{N}$, and prepared in state $|m\rangle$ at $t=0$, the retarded Green's function $G_{m n}(t)$ gives the complex amplitude of probability to measure the system in state $|n\rangle$ at time $t[50,52]$. In particular, $G_{m m}(t)$ measures the coherence of each state $|m\rangle$ over time. Given those two subfamilies of Green's functions (response functions in optics, propagation functions in solid state physics), our theoretical approach aims to connect both through loop Feynman diagrams.

The formalism of Green's functions is a very general way to propagate physical quantities in space and time. As such, they have a variety of applications: for example, another subfamily is used in surface optics and multilayer systems, where they help solving Maxwell equations with boundary conditions and transfer the local optical response to the far field [54]. Here we calculate the local response, so this third subfamily is not relevant.

Matsubara frequency space and second quantization. For convenience, we choose to handle imaginary-time Green's functions $\mathfrak{G}_{m n}$ (whose argument is a complex number within $\mathbb{C}-\mathbb{R}$ ) instead of $G_{m n}$ (whose argument is a real number) $[50,52]$. In frequency domain, both are related through analytical continuity and then constitute two equivalent mathematical pictures [52]:

$$
G_{m n}(\omega)=\mathfrak{G}_{m n}\left(\omega+\imath 0^{+}\right)
$$

where $\imath 0^{+}$represents a small positive imaginary part. Eventually, it will account for the finite widths of the transitions between states. Given the eigenfrequencies $\omega_{m}$ associated to the eigenstates $|m\rangle$ of a system, the imaginary-time Green's functions read [50, 52]:

$$
\mathfrak{G}_{m n}(z)=\frac{\delta_{m n}}{z-\omega_{m}},
$$

with $z \in \mathbb{C}-\mathbb{R}$. The advantage of $\mathfrak{G}_{m n}$ lies, first, in its minimalist expression and, second, in the possibility to apply the residue theorem to simplify the calculations of loop Feynman diagrams (Appendix A). This simplification is made possible because the imaginary-time Green's functions involved in the expansions of optical response functions are computed over the Matsubara frequencies $\imath \omega_{\nu}, \nu \in \mathbb{Z}$, which are the poles of the Fermi-Dirac function $\rho(z)=\left(1+e^{\hbar b z}\right)^{-1}, z \in \mathbb{C}$ and $b=1 / k_{B} T$ (Appendix A). This formalism is directly linked to the concepts of fermion creation and annihilation operators, $c_{m}^{\dagger}$ and $c_{m}$, in second quantization. Indeed, equation (7) is equivalent to the knowledge of the diagonal hamiltonian $\mathcal{H}_{0}$ of the system:

$$
\mathcal{H}_{0}=\sum_{m} \hbar \omega_{m} c_{m}^{\dagger} c_{m}
$$


Without perturbation, each state $|m\rangle$ freely propagates with the probability amplitude $\mathfrak{G}_{m m}\left(\omega+\imath 0^{+}\right)$. But when an external field $\mathbf{E}$ interacts with the system, $|m\rangle$ may be annihilated (via $c_{m}$ ) to the benefit of another state $|n\rangle$ (created via $c_{n}^{\dagger}$ ). This actually translates the promotion of the system from state $|m\rangle$ to state $|n\rangle$ by absorption of light, or its relaxation by emission of light. Such a perturbation is necessarily driven by a perturbative hamiltonian with cross-terms $c_{n}^{\dagger} c_{m}$. This is indeed the case of light-matter interaction, based on the dipolar hamiltonian:

$$
\mathcal{H}_{\mathrm{LM}}=-\mathbf{p} \cdot \mathbf{E},
$$

wherein the dipole moment of the system reads:

$$
\mathbf{p}=\sum_{n, m} \mathbf{p}_{n m} c_{n}^{\dagger} c_{m}
$$

Since the electric field is quantized via boson operators $a^{(i)} / a^{(i)^{\dagger}}$ associated to $i$-polarized photons [55], the interaction hamiltonian $\mathcal{H}_{\mathrm{LM}}$ involves 3-particle terms of the forms $p_{n m}^{i} c_{n}^{\dagger} c_{m} a^{(i)}$ (absorption) and $p_{m n}^{i} c_{m}^{\dagger} c_{n} a^{(i)^{\dagger}}$ (emission). For instance, the first term (absorption) tells us that the excitation of the system by light disturbs the propagation of state $|m\rangle$ by changing the system into state $|n\rangle$ with the probability amplitude $p_{n m}^{i}$. It translates the annihilations of state $|m\rangle$ and photon $i$ (via $\left.c_{m} a^{(i)}\right)$, and the creation of state $|n\rangle$ (via $c_{n}^{\dagger}$ ).

Drawing a loop Feynman diagram then consists in: first, associating oriented lines to the quantum states of the system when they freely propagate with the probability $\mathfrak{G}_{m m}\left(\omega+\imath 0^{+}\right)$and, second, connecting two quantum states with a photon (represented as a wave) in a vertex when the system interacts with light with the probability $p_{n m}^{i}$. Figure 2 a gives such a graphical representation of the SFG process for a simple system. This loop diagram is made of three oriented propagators and three light-matter vertices (each vertex is a 3-particle node, i.e. between two quantum states and a photon). Thanks to this diagrammatic representation, it is then possible to apply the Feynman rules for calculating optical response functions.

Implementation of the method. The use of Feynman diagrams for the analytical computation of the linear polarizability $\boldsymbol{\alpha}$ and the hyperpolarizabilities $\boldsymbol{\beta}$ and $\boldsymbol{\gamma}$ of a simple or a composite system relies on twelve steps $[50,52]$ (some quantities, like the virtual bosons which translate energy transfers between two subsystems, Figure 3, will be defined in the next part):

1) Define the system by its number $N_{l}$ of partners (or subsystems); define the optical process by the number $N_{p}$ of photons involved, their nature (creation or annihilation) and their frequency relationships (e.g. $\omega_{3}=\omega_{1}+\omega_{2}$ for SFG); first-order $\boldsymbol{\alpha}$ functions are represented by diagrams for which $N_{p}=2$, second-order $\boldsymbol{\beta}$ functions when $N_{p}=3$, and third-order $\gamma$ functions when $N_{p}=4$;
2) Define the total number $N_{v}$ of interaction processes, first, between the partners and the photons $\left(N_{p}\right)$ and, second, between the partners themselves $\left(N_{v}-N_{p}\right)$, reminding that two nodes linked by a virtual boson propagator represent one interaction process;

3) Draw all the topologically distinct loop diagrams made of $N_{l}$ loops (as many as subsystems) and $N_{v}$ interaction processes;

4) For each loop, assign an implicit Matsubara frequency (e.g. $\left.\imath \omega_{\nu}\right)$ to the propagator associated to the initial state;

5) Apply the energy conservation rule (in terms of frequencies) at each vertex with boson frequencies chosen among those present in the system, by ensuring that the constitutive energy relationship (e.g. $\omega_{3}=\omega_{1}+\omega_{2}$ ) applies at one and only one vertex; draw as many distinct diagrams as possible by considering all allowed frequencies, initial states and directions of rotation on the loops; if some diagrams are equivalent, keep only one of them;

6) Determine for each vertex the coupling constant corresponding to the interaction hamiltonian (e.g. $p_{n m}^{i}$ for light-matter interaction) and multiply them;

7) Determine for each propagator the associated imaginary-time Green's function and multiply them;

8) Multiply by $(-1)^{N_{p}+1} \cdot b^{N_{p}-N_{v}-1} \cdot \hbar^{N_{p}-2 N_{v}}$, where $b=1 / k_{B} T$;

9) Sum over all the quantum numbers and all the implicit Matsubara frequencies to get the response function with imaginary frequency arguments;

10) Use the residue theorem (Appendix A) in order to reduce the sums over the implicit frequencies;

11) Replace the imaginary frequencies $\imath \omega$ of photons by $\omega+\imath 0^{+}$to get the response function with real frequency arguments;

12) Introduce the damping constants $\Gamma_{n m}$ by replacing each term $\left( \pm \omega_{n m}+\imath 0^{+}\right)$by $\left( \pm \omega_{n m}+\imath \Gamma_{n m}\right)$. This last rule takes into account the finite lifetimes of real quantum states.

Along steps 1 to 9, the calculations involve imaginarytime Green's functions and Matsubara frequencies $\imath \omega_{\nu}$. As optical response functions are defined with real frequencies, the analytical continuity depicted by equation (6) is used at step 11 to deduce the useful response functions $\boldsymbol{\alpha}(\omega), \boldsymbol{\beta}\left(\omega_{1}, \omega_{2}\right)$ and $\boldsymbol{\gamma}\left(\omega_{1}, \omega_{2}, \omega_{3}\right)$.

Illustration in the case of simple systems. According to these rules, the linear polarizability $\boldsymbol{\alpha}$ of a simple system (i.e. made of one loop) coincides with the usual formula 


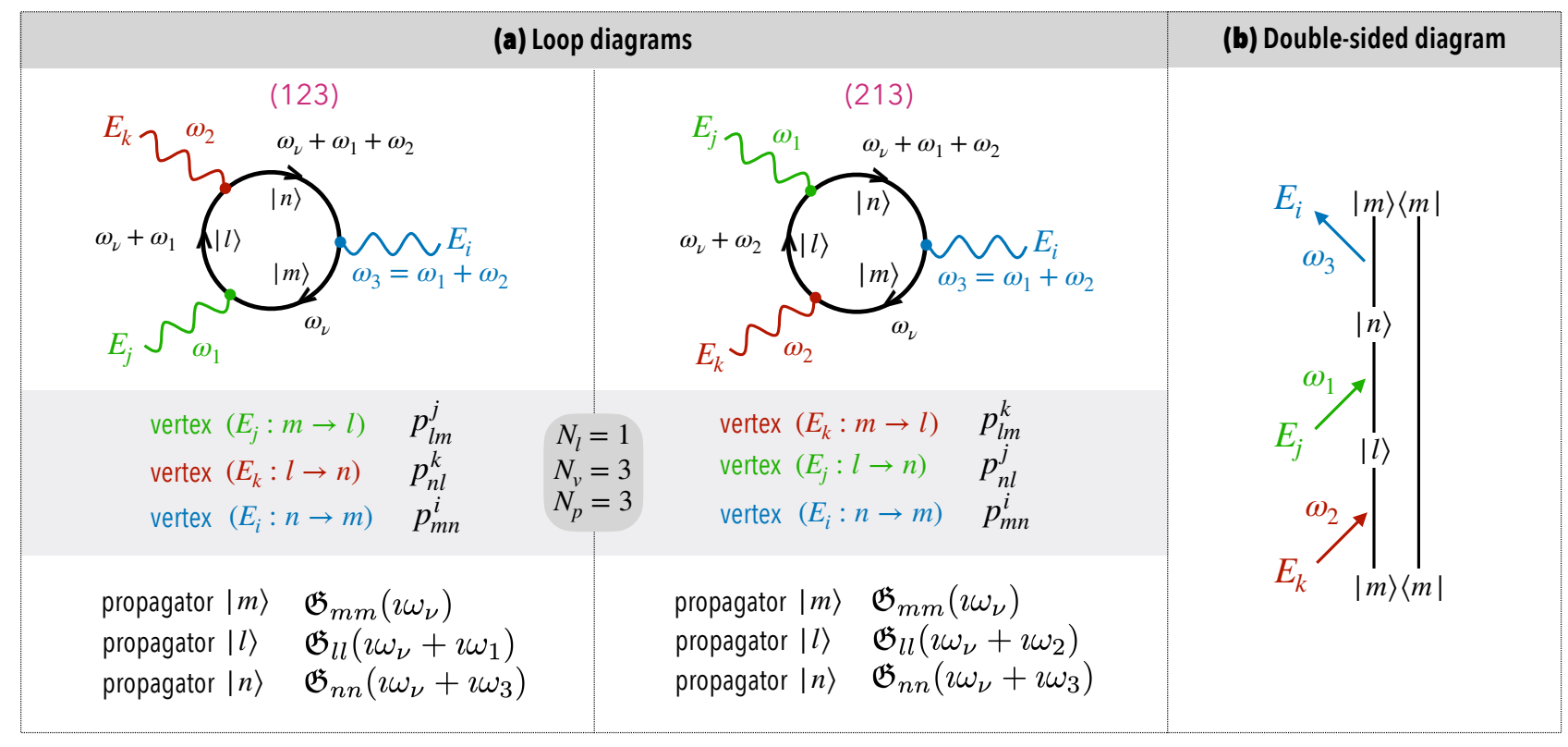

FIG. 2. Feynman diagram for sum-frequency generation in simple systems. a, We draw diagrams made of a single loop $\left(N_{l}=1\right)$, which embody the hyperpolarizability $\beta_{i j k}\left(\omega_{1}, \omega_{2}\right)$ of a simple system. The oriented lines correspond to the propagators of its quantum states, labelled by $m, n$ and $l$. The waves represent the three photons $\left(N_{p}=3\right)$ involved in the SFG process, at frequencies $\omega_{1}, \omega_{2}$ and $\omega_{3}$. At each vertex between a wave and two propagators, the total frequency is conserved (Feynman rule \#5). For the three vertices $\left(N_{v}=3\right)$, the coupling constants correspond to the appropriate components of the transition dipole moment $\mathbf{p}$, as listed on the right (Feynman rule \#6). For the three propagators, the corresponding imaginary-time Green's functions are listed too (Feynman rule \#7). These two diagrams lead to equations (12-13). b, Conventional double-sided Feynman diagram, commonly used in nonlinear optics.

$[46,47,56,57]:$

$$
\begin{aligned}
& \alpha_{i j}(\omega)= \\
& \sum_{m, n} \frac{\rho\left(\omega_{m}\right)}{\hbar}\left(\frac{p_{n m}^{i} p_{m n}^{j}}{\omega+\omega_{n m}+\imath \Gamma_{n m}}-\frac{p_{n m}^{j} p_{m n}^{i}}{\omega-\omega_{n m}+\imath \Gamma_{n m}}\right) .
\end{aligned}
$$

as derived in Appendix B. At this stage, it evidences the relevance of the diagrammatic method but we rather dwell on the computation of the hyperpolarizability $\boldsymbol{\beta}$ to fully illustrate the mechanism of our approach. For this purpose, we need to consider the two loop diagrams drawn in Figure 2a, which result from the application of Feynman rules \#1 to \#5 with three photons and $\omega_{3}=\omega_{1}+\omega_{2}$. They embody the SFG process occurring through a single loop system, when two input photons of frequencies $\omega_{1}$ and $\omega_{2}$ interact with it. These photons are respectively $j$ - and $k$-polarized, while the resulting SFG photon is considered $i$-polarized. Actually, we are sure that there are only two relevant diagrams: there are as many 1-loop diagrams as cyclic permutations in $\{1,2,3\}$, that is $(3-1) !=2$. This is why we name them (123) and (213) in Figure 2a, referring to the corresponding cyclic permutations of photons. Performing this cyclic permutation is graphically equivalent to changing the direction of rotation or inverting $\omega_{1}$ and $\omega_{2}$ on this loop.

By applying the first nine Feynman rules to these two diagrams, we obtain (in terms of imaginary frequencies):

$$
\begin{aligned}
& \beta_{i j k}^{(123)}\left(\imath \omega_{1}, \imath \omega_{2}\right)=\frac{(-1)^{4}}{b \hbar^{3}} \sum_{n, m, l} p_{m n}^{i} p_{n l}^{k} p_{l m}^{j} \\
& \sum_{\nu} \mathfrak{G}_{m m}\left(\imath \omega_{\nu}\right) \mathfrak{G}_{l l}\left(\imath \omega_{\nu}+\imath \omega_{1}\right) \mathfrak{G}_{n n}\left(\imath \omega_{\nu}+\imath \omega_{3}\right),
\end{aligned}
$$

and:

$$
\begin{aligned}
& \beta_{i j k}^{(213)}\left(\imath \omega_{1}, \imath \omega_{2}\right)=\frac{(-1)^{4}}{b \hbar^{3}} \sum_{n, m, l} p_{m n}^{i} p_{n l}^{j} p_{l m}^{k} \\
& \sum_{\nu} \mathfrak{G}_{m m}\left(\imath \omega_{\nu}\right) \mathfrak{G}_{l l}\left(\imath \omega_{\nu}+\imath \omega_{2}\right) \mathfrak{G}_{n n}\left(\imath \omega_{\nu}+\imath \omega_{3}\right) .
\end{aligned}
$$

The Appendix $\mathrm{C}$ details the derivation of both quantities and the application of rule \#10. Applying rules \#11 and \#12 to Eq. (46) and (47), and considering the formal equivalence between the Fermi-Dirac distribution $\rho\left(\omega_{l}\right)$ and the density matrix $\hat{\rho}_{l l}$ (as shown in Appendix D), we 
get:

$$
\begin{aligned}
& \beta_{i j k}^{(123)}\left(\omega_{1}, \omega_{2}\right)= \\
& \frac{1}{\hbar^{2}} \sum_{m, n, l} \hat{\rho}_{l l}\left[\frac{p_{l n}^{i} p_{m l}^{j} p_{n m}^{k}}{\left(\omega_{n l}-\omega_{3}-\imath \Gamma_{n l}\right)\left(\omega_{m l}-\omega_{1}-\imath \Gamma_{m l}\right)}\right. \\
& +\frac{p_{n m}^{i} p_{l n}^{j} p_{m l}^{k}}{\left(\omega_{n m}+\omega_{3}+\imath \Gamma_{n m}\right)\left(\omega_{m l}-\omega_{2}-\imath \Gamma_{m l}\right)} \\
& +\frac{p_{n m}^{i} p_{l n}^{j} p_{m l}^{k}}{\left(\omega_{m n}-\omega_{3}-\imath \Gamma_{m n}\right)\left(\omega_{n l}+\omega_{1}+\imath \Gamma_{n l}\right)} \\
& \left.+\frac{p_{m l}^{i} p_{n m}^{j} p_{l n}^{k}}{\left(\omega_{m l}+\omega_{3}+\imath \Gamma_{m l}\right)\left(\omega_{n l}+\omega_{2}+\imath \Gamma_{n l}\right)}\right]
\end{aligned}
$$

Here, we use the same labels (a1), (b2), etc. as employed by R.W. Boyd [46] in his equation (3.6.18) to show that our loop diagrams provide an equivalent result as the doubled-sided ones, such as that drawn in Figure $2 \mathrm{~b}$. However, eight double-sided diagrams are necessary to account for the complete hyperpolarizability, whereas only two loop diagrams suffice, meaning that a 1-loop diagram contains as much information as four double-sided ones. This constitutes a great advantage of our graphical formalism, much more compact. Besides, for the computation of third-order hyperpolarizabilities $\gamma_{i j k l}$ in a single loop system, we only need $(4-1) !=6$ loop diagrams (as many as cyclic permutations in $\{1,2,3,4\}$ ) instead of 48 double-sided diagrams [46]. Finally, our formalism allows to tackle systems characterized by continuous statistical distributions $\rho(\omega)$ (e.g. metals) at any temperature, which is not possible with the formalism based on the density matrix picture. Conversely, following the equivalence shown in Appendix D, the loop-diagram method is indeed more universal as it also applies to discrete systems like molecules or atoms, allowing to recover the classical density matrix formulation [46] (which appears here as a special case of the Green-Matsubara formalism).

Eventually, in the case of a purely molecular system (of dipole moment $\boldsymbol{\mu}$ ) probed by IR-vis SFG spectroscopy, the IR-resonant response eventually yields, as detailed in
Appendix C:

$$
\begin{aligned}
\beta_{i j k}\left(\omega_{1}, \omega_{2}\right) & =\beta_{i j k}^{(123)}\left(\omega_{1}, \omega_{2}\right)+\beta_{i j k}^{(213)}\left(\omega_{1}, \omega_{2}\right) \\
& =\frac{1}{\hbar} \sum_{v} \frac{\partial_{v} \alpha_{i j}\left(\omega_{3}\right) \partial_{v} \mu_{k}}{\omega_{2}-\omega_{v}+\imath \Gamma_{v}}
\end{aligned}
$$

where the partial derivative along each normal coordinate $Q_{v}$ is given by:

$$
\partial_{v}=\sqrt{\frac{\hbar}{2 \omega_{v}}} \frac{\partial}{\partial Q_{v}} .
$$

We actually retrieve the usual formula for IR-resonant molecular systems $[12,58]$.

At this stage, in order to understand how the nonlinear response of a molecular system is modified by the presence of a partner (e.g. substrate, nanoparticle), we propose to build new diagrams made of two loops (for both the molecule and the partner) connected by new interaction vertices. These are expected to account for the coupling between the two interacting subsystems and to enable us to treat the complex case of composite systems.

\section{DIAGRAMMATIC THEORY OF BIPARTITE COMPOSITE SYSTEMS}

In this section, we dwell on the specific case of composite systems made of two subsystems, and focus on the second order response. In order to give the broadest scope to our method and make it as general as possible, we elaborate on the whole reasoning on a mathematical point of view. In particular, we enumerate all the possible bipartite diagrams, which leads us to more than thirty. This may seem high, but most of these are not relevant to calculate meaningful physical quantities. The results are quite simplified thanks to the use of the twelve Feynman rules and a selection criterion, introduced hereafter, to keep only relevant diagrams for a particular situation. Applying these rules, it becomes rather simple to draw and calculate the relevant diagrams without concentrating on the upstream derivation they arise from. This section can be considered as a rigorous demonstration leading to practical and operative results, as illustrated in the last sections.

Interactions between subsystems. In the case of bipartite systems (e.g. made of a substrate and a molecule), the bulk of the challenge lies in finding the vertex accounting for the interaction between the two partners. This amounts to finding the right interaction hamiltonian $\mathcal{H}_{\text {int }}$ in terms of fermion operators. Without loss of generality, it is always possible to expand a two body interaction as [50, 52]:

$$
\mathcal{H}_{\mathrm{int}}=\sum_{n, m} \sum_{r, s} C_{n m}^{r s} c_{n}^{\dagger} d_{r}^{\dagger} c_{m} d_{s},
$$




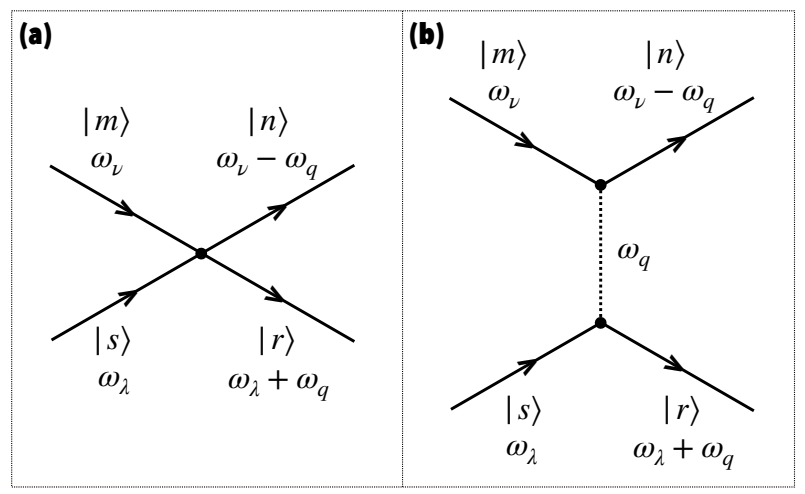

FIG. 3. Four-particle vertex. a, Diagrammatic representation of the interaction vertex associated to equation (16) as a node between four propagators. Each subsystem is characterized by its own implicit Matsubara frequency, $\omega_{\nu}$ or $\omega_{\lambda}$. b, Decomposition of a 4-particle vertex into two 3-particle nodes. This representation introduces a virtual boson (which may transfer energy $\omega_{q}$ ) and allows splitting the system into two distinct subsystems.

wherein $c_{n} / c_{n}^{\dagger}$ and $d_{r} / d_{r}^{\dagger}$ are the fermion operators associated to the two subsystems, respectively. The quantity $C_{n m}^{r s}$ is nothing but the coupling constant which intervenes explicitly into the computation of hyperpolarizabilities. In the case of a dipolar coupling between two dipole moments $\mathbf{p}$ and $\boldsymbol{\mu}$ with a distance $r$ :

$$
C_{n m}^{r s}=\sum_{k l} W_{k l}(r) p_{n m}^{k} \mu_{r s}^{l}
$$

where $\boldsymbol{W}(r)$ is the dipole-dipole interaction matrix encoding the $1 / r^{3}$ spatial dependence of the coupling. In the case of an electrostatic interaction between a dipole moment $\boldsymbol{\mu}$ and a static electric field $\mathbf{E}_{0}$ :

$$
C_{n m}^{r s}=-\delta_{m 0} \delta_{n 0} \sum_{l} \mu_{r s}^{l} E_{0}^{l}
$$

At this stage, however, we propose to treat the interactions on a very general point of view. Equation (16) means that such interactions take the form of 4-particule vertices (via $c_{n}^{\dagger} d_{r}^{\dagger} c_{m} d_{s}$ ), as pictured in Figure 3a, while light-matter interaction involves 3 -particle vertices. For clarity, it is possible to decompose a 4-particle vertex into two 3-particle nodes (Figure 3b). For that purpose, we formally introduce a virtual boson which conveys an exchange of energy or a transfer of quantum numbers. This virtual boson is then represented as a dashed line. In the case of electromagnetic interactions, as in Eq. (17) and (18) for which dipoles and fields exchange energy quanta corresponding only to their oscillating frequencies, the frequencies of the bosons must match one of those present in the system (hence rule \#5). The choice of this representation facilitates the counting of bipartite diagrams that it is possible to generate.
Enumeration of bipartite diagrams. The hardest task may be to satisfy Feynman rule \#3 by determining the full set of topologically distinct diagrams. A list of 2loop diagrams representing substrate/molecule systems is given in Figure 4. Here, the distinction between 'substrate' and 'molecule' is purely illustrative and exemplary. This list is totally general for any bipartite system with the result that Figure 4 inventories all the SFG diagrams we can imagine to couple two interacting subsystems thanks to one and two virtual bosons. To demonstrate it, we split our reasoning into three parts. First, we assume that the three photons are equivalent and indistinguishable. In this case, the enumeration consists in determining how many possibilities there are to place propagators and vertices on two loops, without regard for nature of the vertices (light-matter or matter-matter). In a way, this boils down to enumerating the skeletons of the 2-loop SFG diagrams (bare diagrams in Figure 4). Second, we distinguish the three photons and count how many cyclic permutations it is possible to generate for each bare diagram (enumeration in Figure 4). Third, this allows labelling the three light-matter vertices by $\omega_{1}, \omega_{2}$ or $\omega_{3}$, drawing the virtual bosons which bridge the two loops and establishing the complete list of diagrams on Figure 4.

These three steps illustrated in Figure 4 are governed by several relationships between the four parameters $N_{\text {mol }}, N_{\text {sub }}, V$ and $P$, standing for the number of molecular propagators, the number of propagators associated to the substrate, the number of virtual bosons (i.e. 4particle vertices) and the number of photons interacting with the molecule, respectively.

To start, we can notice that the total number of propagators is fixed for a given number of 4-particle vertices:

$$
N_{\text {sub }}+N_{\text {mol }}=3+2 V .
$$

Indeed, there are as many propagators $\left(N_{\text {sub }}+N_{\text {mol }}\right)$ as nodes: as each virtual boson counts for 2 nodes, and as there are 3 photons interacting with the whole system, the total number of nodes is equal to $3+2 V$. Besides, a diagram built with $V$ virtual bosons requires at least $V$ molecular propagators and $V$ propagators for the substrate: $N_{\mathrm{sub}}, N_{\mathrm{mol}} \geqslant V$. From equation (19), we deduce that:

$$
V \leqslant N_{\text {mol }}, N_{\text {sub }} \leqslant V+3 .
$$

As a consequence, at a fixed $V$, we always count four possible couples $\left(N_{\mathrm{mol}}, N_{\mathrm{sub}}\right)$, which are symmetric according to the permutation ( $\mathrm{mol} \leftrightarrow \mathrm{sub})$ :

$$
\begin{array}{ll}
(V+3, V), & (V+2, V+1), \\
(V, V+3), & (V+1, V+2) .
\end{array}
$$

The first step of diagram building thus comes down to examining the only two cases with $(V+3, V)$ and $(V+2, V+1)$ to create the bare diagrams. 


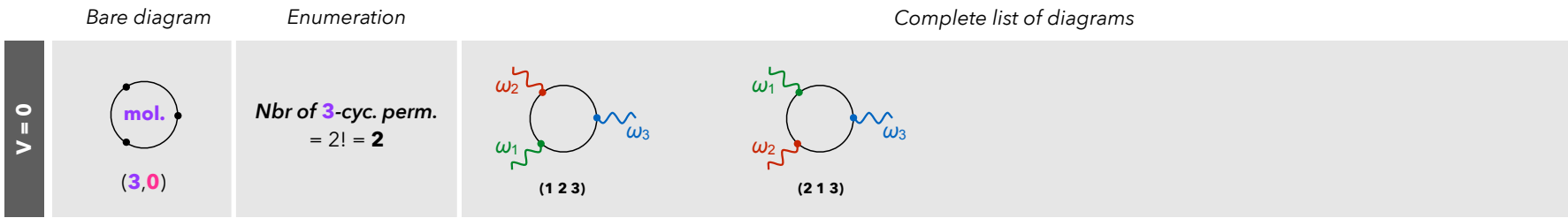

(5br of 1-cyc. perm.
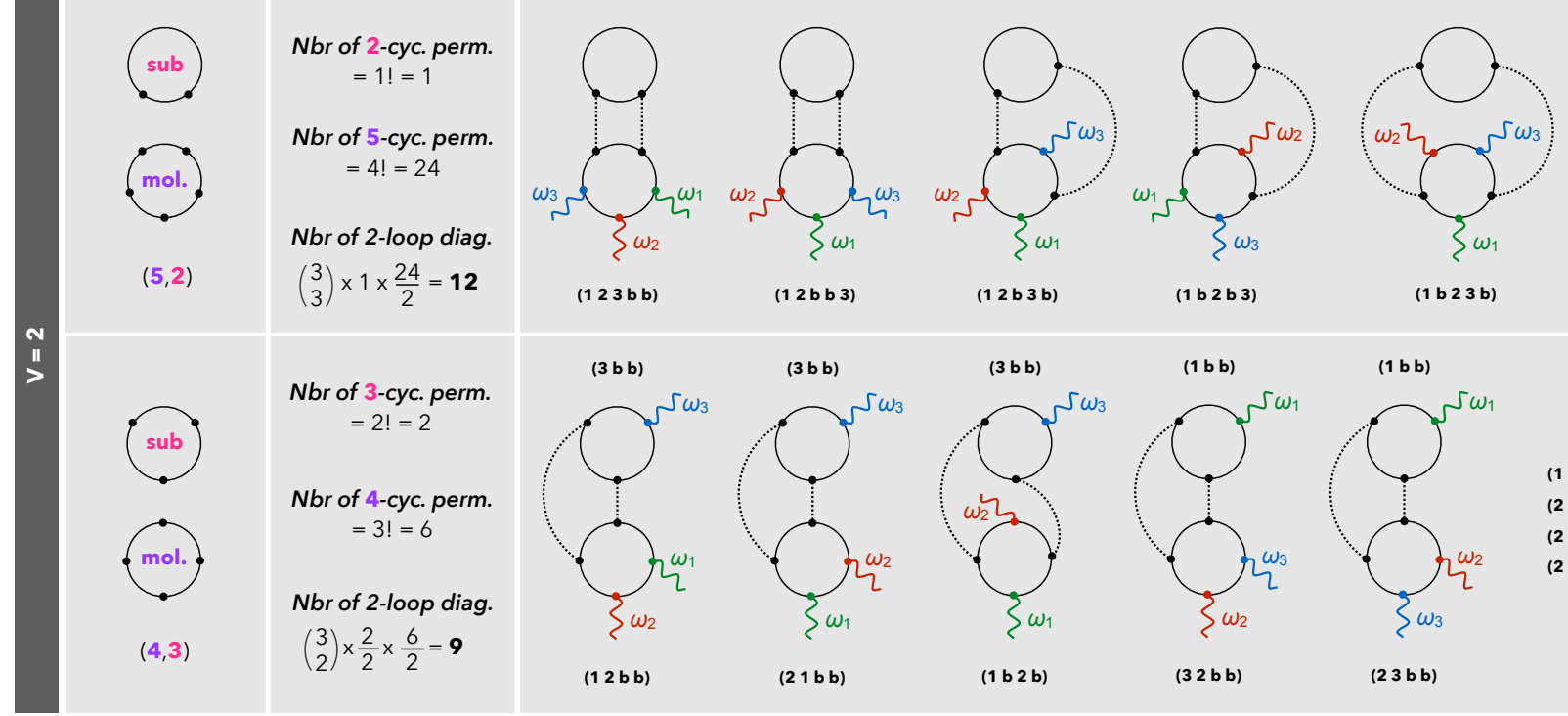

(1 3 b b 2)

(1 3 b 2 b)

(1 $32 \mathrm{~b} \mathrm{~b}$ )

(1 b 3 b 2)

(1 b 32 b)

(1 b b 2 3)

(1 $23 \mathrm{~b}$ b)

(1 2 b b 3)

(1 2 b 3 b)

(1 b 2 b 3)

(1 b 23 b)

(1 b b 32 )

(3 b b)

sub

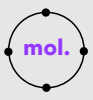

$(4,3)$

Nbr of 3-cyc. perm.

$$
=2 !=2
$$

Nbr of 4-cyc. perm.

$$
=3 !=6
$$

Nbr of 2-loop diag.

$\left(\begin{array}{l}3 \\ 2\end{array}\right) \times \frac{2}{2} \times \frac{6}{2}=9$

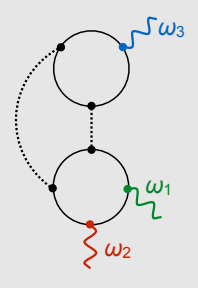

$$
\text { (3bb) }
$$
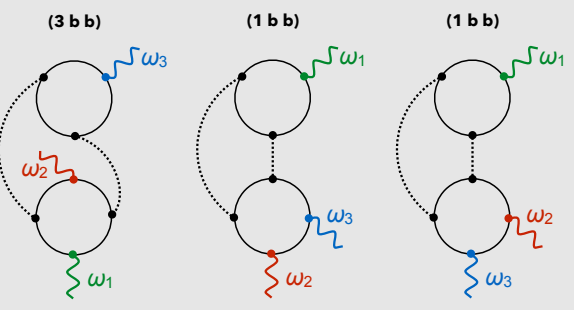

(1 b b) - (2 b 3 b)

(2 bb) - (1 $3 b b)$

(2 b b) - (3 1 b b)

(1 2 b b)

(2 1 b b)

(1 b 2 b)

(3 2 b b)

(2 3 b b)

FIG. 4. Bipartite diagrams. The Feynman diagrams that it is possible to generate for bipartite systems, like substrate/molecule interfaces, are classified according to the number $N_{\text {sub }}$ of propagators associated to the substrate (upper loop), the number $N_{\text {mol }}$ of molecular propagators (lower loop), and the number $V$ of virtual bosons (dashed lines). In the first column, we give the bare diagrams for the different couples $\left(N_{\text {mol }}, N_{\text {sub }}\right) \in\{(3,0) ;(4,1) ;(3,2) ;(5,2) ;(4,3)\}$. They only exhibit propagators and nodes, without regard for the nature of the vertices it is possible to assign to these nodes. In the second column, we enumerate the cyclic permutations of $N_{\text {sub }}$ and $N_{\text {mol }}$ elements and deduce the total number of 2-loop diagrams. This actually illustrates the demonstration of equations (22) and (23). In the third column, we explicitly draw the corresponding diagrams, named by their associated cyclic permutations. These permutations refer to the sequences of photons and virtual bosons, $\{1,2,3\} \cup\{\mathrm{b}\}^{V}$, along the two oriented loops. Let us notice that this figure only gives a half of the bipartite diagrams: the second part is obtained by inverting the two subsystems (mol $\leftrightarrow$ sub). 


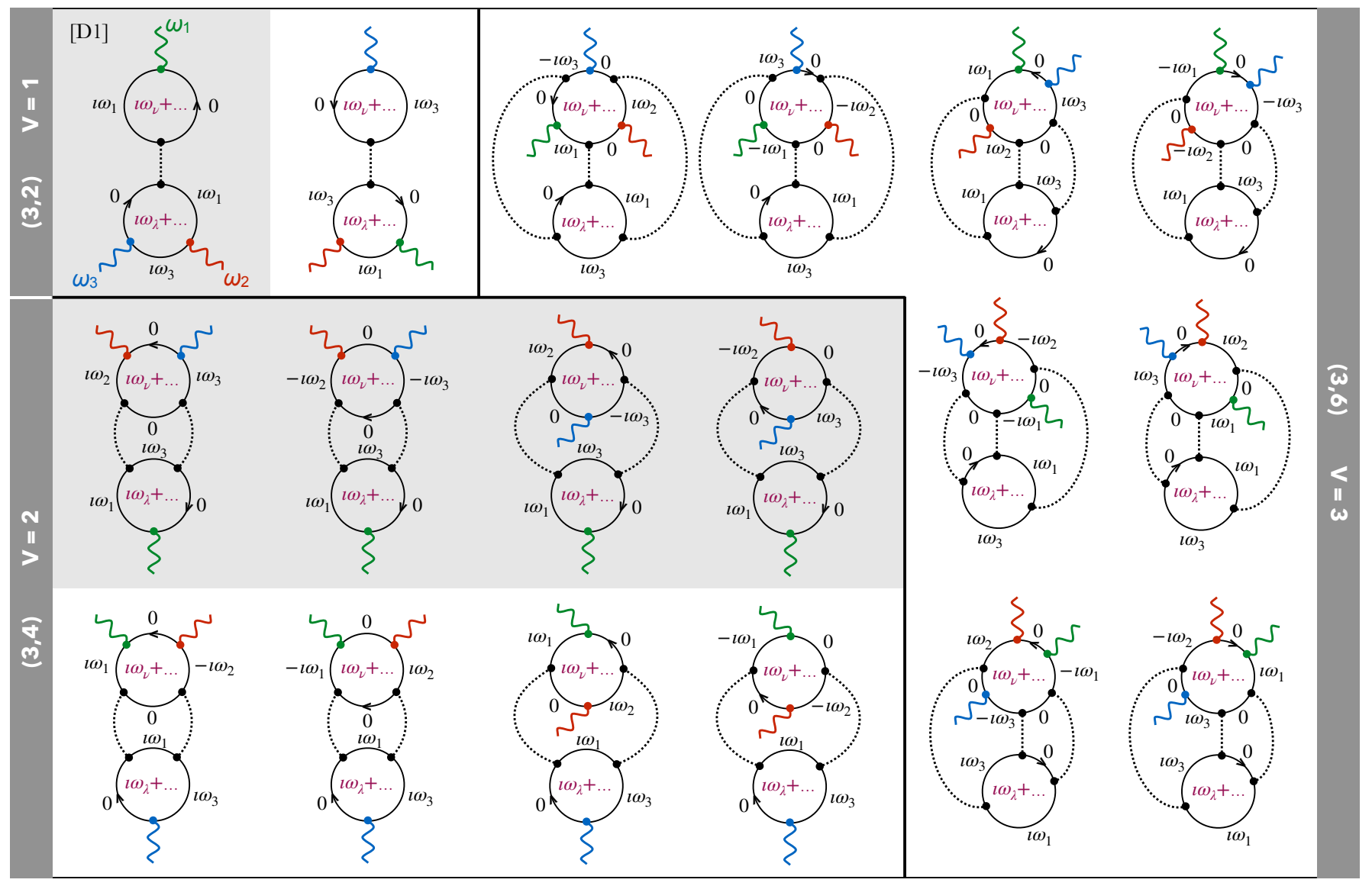

FIG. 5. Second-order molecular response. List of the 2-loop diagrams involved in the second-order molecular response of any bipartite system and belonging to $(3,2),(3,4)$ and $(3,6)$ families (see Figure 2 for the $(3,0)$ diagrams). Five additional diagrams, deduced from those in the grey zone by exchanging $\omega_{1}$ and $\omega_{2}$, must be added to complete the list to 23 . The 23 other diagrams (not shown) are deduced by changing the direction of rotation on the molecular loop. Each propagator is calculated with an imaginary frequency added to the Matsubara frequency $\left(\imath \omega_{\nu}\right.$ for the upper loop and $\imath \omega_{\lambda}$ for the lower loop, as recalled inside both). The green, red and blue waves respectively correspond to the input photons $\omega_{1}$ and $\omega_{2}$, and the output photon $\omega_{3}$. The arrows indicate the initial states (i.e. propagators at Matsubara frequency). The sum of all these contributions gives access to the total hyperpolarizability $\beta_{i j k}\left(\omega_{1}, \omega_{2}\right)$ of a molecule, associated to the lower loop, under the influence of a partner (e.g. substrate, nanoparticle), associated to the upper loop.

In order to start decorating the bare diagrams with photons and bosons, in other words to assign their vertices, we note that the number $P$ of photons which interact with the molecule is determined by the two numbers $V$ and $N_{\text {mol }}$ :

$$
P=N_{\mathrm{mol}}-V .
$$

Enumeration starts by choosing a way to assign $P$ photons to the molecular loop among the three photons involved in the second-order process. In other words, the number of possible 2-loop diagrams with all photons assigned to a loop simply corresponds to the value of the binomial coefficient $\left(\begin{array}{l}3 \\ P\end{array}\right)$. Consequently, there is a single possible photon-assigned diagram for the first couple $(V+3, V): P=3$ and $\left(\begin{array}{l}3 \\ 3\end{array}\right)=1$. In the case of the second couple $(V+2, V+1), P=2$ and leads to three photonassigned diagrams: $\left(\begin{array}{l}3 \\ 2\end{array}\right)=3$. At this stage, the natures of all vertices on each loop are known because the virtual bosons $\{b\}$ are indeed indistinguisable (their energies will be determined later on by rule \#5). The set of photons is noted $\{1,2,3\}$ and the set of virtual bosons $\{b\}^{V}$. For each loop made of $k$ propagators (and then $k$ nodes), we assign a cyclic permutation of order $k$ representing the cyclic sequence of vertices (photons and virtual bosons) along the oriented loop. For instance, in the case of the 1-loop diagrams of simple systems (Figures 2a and 4), we count two diagrams assigned to the permutations (123) and (213). For bipartite systems, this logic has to be applied to both loops. Since there are $(k-1)$ ! distinct cyclic permutations for a fixed order $k$, we deduce that there are $\left(N_{\text {mol }}-1\right)$ ! and $\left(N_{\text {sub }}-1\right)$ ! possibilities for the molecular and substrate loops, respectively. Due to the indistinguishability of the virtual bosons, these numbers have to be divided by $V$ ! (when $N_{\mathrm{mol} / \mathrm{sub}}>V$ ) to get the 
number of decorated diagrams, assuming a fixed photon assignment.

Specifically, in the first case with $\left(N_{\text {mol }}, N_{\text {sub }}\right)=$ $(V+3, V)$, the substrate loop exhibits as many nodes as virtual bosons $\left(N_{\text {sub }}=V\right)$. Hence, the three photons only connect with the molecule loop, so that a unique cyclic permutation of order $N_{\text {mol }}=V+3$ is required to tag the 2-loop diagrams. We deduce that there are $(V+2)$ ! distinct diagrams here, except for the virtual bosons indistinguishability. Eventually, the total number of 2-loop diagrams associated to the couple $(V+3, V)$ reads:

$$
\left(\begin{array}{l}
3 \\
3
\end{array}\right) \times \frac{(V+2) !}{V !}=V^{2}+3 V+2 .
$$

In Figure 4, we indeed draw 6 diagrams for $V=1$ and 12 diagrams for $V=2$. These are respectively described by permutations of types $(123 \mathrm{~b})$ and $(123 \mathrm{bb})$ on the molecular loop. In the second case with $\left(N_{\text {mol }}, N_{\text {sub }}\right)=$ $(V+2, V+1)$, both the substrate and the molecule loops are connected with photons, and each diagram is thus described by its own cyclic permutations. With the same reasoning, the total number of such 2-loop diagrams is given by:

$$
\left(\begin{array}{l}
3 \\
2
\end{array}\right) \times \frac{V !}{V !} \times \frac{(V+1) !}{V !}=3 V+3 .
$$

In Figure 4, we actually draw 6 diagrams for $V=1$ and 9 diagrams for $V=2$. They respectively correspond to permutations of types $(1 \mathrm{~b}) \cdot(23 \mathrm{~b})$ and $(1 \mathrm{bb}) \cdot(23 \mathrm{bb})$ on the substrate and molecular loops, respectively. As a matter of fact, taking the mol $\leftrightarrow$ sub symmetry into account, we demonstrate that, for a fixed value of $V \geqslant 1$, there are exactly $2 V^{2}+12 V+10$ distinct 2-loop diagram which address Feynman rule \#3. Besides, our method is constructive: we show how to generate them from cyclic permutations.

The list provided in Figure 4 is then complete for $V \leqslant 2$ and can be extended as explained above for bigger values of $V$. This means that the total $\beta\left(\omega_{1}, \omega_{2}\right)$ of the full system is the sum of all the diagrams drawn with $V \geqslant 0$. However, as we will see below, most of those diagrams are not interesting on a physics point of view. It is indeed, and fortunately, possible to restrict ourselves to fewer diagrams.

\section{Diagrams of interest for 2 nd-order molecular response.}

Amongst the diagrams that we have just enumerated, a selection criterion can be established in order to keep only those involved in the calculation of the second-order hyperpolarizability $\beta_{i j k}\left(\omega_{1}, \omega_{2}\right)$ of a molecule influenced by the presence of a partner, a substrate for example. Indeed, the molecular hyperpolarizability $\beta$ is known to be a 3-rank tensor involving three components of the molecular dipole moment $\boldsymbol{\mu}[46,47,56]$ :

$$
\beta \propto \mu \otimes \mu \otimes \mu
$$

A bipartite diagram can lead to such a 3-component combination if, and only if, the molecular states are described by three propagators: $N_{\text {mol }}=3$. As we intend to compute $\beta_{i j k}\left(\omega_{1}, \omega_{2}\right)$, with the input frequencies $\omega_{1}$ and $\omega_{2}$, the three molecular propagators of each diagram must always be associated to consecutive frequencies of type $\left(0, \imath \omega_{1}, \imath \omega_{3}\right)$ or $\left(0, \imath \omega_{2}, \imath \omega_{3}\right)$ above the Matsubara $\imath \omega_{\lambda}$ frequency. As a consequence, the molecular loop has to be identical, in terms of propagators, to one of those depicted in Fig. 2. In other words, the diagrams relevant for the calculation of a molecular $\beta_{i j k}\left(\omega_{1}, \omega_{2}\right)$ coupled to a partner are those explicitly encompassing one of the 3-propagator loops describing the non-interacting $\beta_{i j k}\left(\omega_{1}, \omega_{2}\right)$.

Using $N_{\text {mol }}=3$, equation (21) thus leads to $0 \leqslant V \leqslant 3$. As a consequence, it is useless to examine bipartite diagrams comprising more than three virtual bosons, so the great majority of the full list of diagrams can be rejected. Under these conditions, we count four couples $\left(N_{\text {mol }}, N_{\text {sub }}\right)$ of interest:

$$
\left\{\begin{array}{c}
N_{\mathrm{mol}}=3 \\
0 \leqslant V \leqslant 3
\end{array}\right\} \quad \Longrightarrow \quad \begin{array}{ll}
(3,0) & (3,4) \\
(3,2) & (3,6)
\end{array}
$$

Applying Feynman rule \#5 (i.e. conservation of energy), it is possible to fill some of these diagrams with several frequency configurations. The diagrams of types $(3,0)$ and $(3,2)$ do not involve more than one virtual boson, so that the associated permutations unambiguously fix the way we can fill them with frequencies. In contrast, when there are two or three virtual bosons, the choice of the relative orientations of the two loops (two possibilities for each loop) gives rise to four possible fillings for each diagram. In Figure 5, we list these filled diagrams, and we outline their essential features here below.

The $(3,0)$ diagrams correspond to the two possible fillings of the 1-loop diagram as explained on Fig. 2 (i.e. inverting $\omega_{1}$ and $\omega_{2}$ or changing the direction of rotation). For all the 2-loop diagrams, we may therefore count the number of possible fillings of the substrate loop, then double the number of diagrams as a consequence of this dual filling on the molecular loop. We count 6 distinct $(3,2)$ diagrams (Fig. 4) corresponding to three choices for the photon interacting with the substrate loop (leading to its filling with frequencies 0 and $\imath \omega_{i}$ above the Matsubara frequency $\imath \omega_{\nu}$, with $\left.i=1,2,3\right)$ and two fillings of the molecular loop. For the $(3,4)$ diagrams, it is possible to check that they correspond to a substrate loop filled with frequencies $\imath \omega_{\nu}+\left(0, \pm \imath \omega_{i}, 0, \pm \imath \omega_{j}\right), i \neq j$, leading to twelve possibilities for this loop, and thus 24 diagrams in total with the molecular loop symmetry. For the $(3,6)$ diagrams, the filling of each substrate loop follows the sequence $\imath \omega_{\nu}+\left(0, \pm \imath \omega_{i}, 0, \pm \imath \omega_{j}, 0, \pm \imath \omega_{k}\right), i \neq j \neq k \neq i$, giving rise to eight possibilities for the upper loop and 16 diagrams as a whole. A total of $48=2+6+24+16$ distinct diagrams must therefore be considered to gather all 


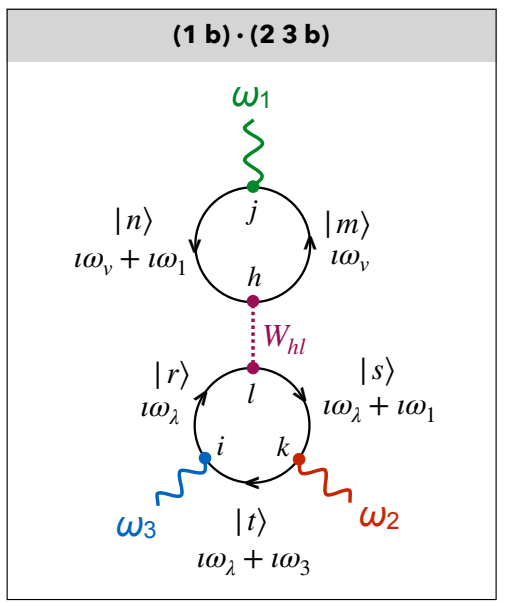

FIG. 6. Example of composite diagram. Filling of the bipartite loop diagram $(1 \mathrm{~b}) \cdot(23 \mathrm{~b})$ used for the derivation of equation (26).

the information of concern when focusing on the secondorder response of a molecule under the influence of its partner, as summarized in Figure 5.

Let us consider the first diagram, named [D1] in Figure 5, whose filling is detailed in Figure 6. Assuming a dipolar coupling driven by equation (17), the application of the first nine Feynman rules leads to:

$$
\begin{aligned}
& \beta_{i j k}^{[\mathrm{D} 1]}\left(\imath \omega_{1}, \imath \omega_{2}\right)=\sum_{h, l} W_{l h} \\
& \times \frac{1}{b \hbar^{2}} \sum_{m, n ; \nu} p_{m n}^{h} p_{n m}^{j} \mathfrak{G}_{m m}\left(\imath \omega_{\nu}\right) \mathfrak{G}_{n n}\left(\imath \omega_{\nu}+\imath \omega_{1}\right) \\
& \times \frac{1}{b \hbar^{3}} \sum_{r, s, t ; \lambda} \mu_{r t}^{i} \mu_{s r}^{l} \mu_{t s}^{k} \mathfrak{G}_{r r}\left(\imath \omega_{\lambda}\right) \mathfrak{G}_{r r}\left(\imath \omega_{\lambda}+\imath \omega_{1}\right) \mathfrak{G}_{t t}\left(\imath \omega_{\lambda}+\imath \omega_{3}\right) .
\end{aligned}
$$

From equation (12) and Appendix B (equation 38), this reads:

$$
\beta_{i j k}^{[\mathrm{D} 1]}\left(\omega_{1}, \omega_{2}\right)=-\sum_{h, l} W_{l h} \alpha_{h j}^{\mathrm{sub}}\left(\omega_{1}\right) \cdot \beta_{i l k}^{(123)}\left(\omega_{1}, \omega_{2}\right),
$$

where $\boldsymbol{\alpha}^{\text {sub }}$ depicts the linear polarizability of the substrate. Here again, a second contribution [D1'] must be considered, by changing the direction of rotation on the molecular loop of [D1]. We thus find the same result with $\beta_{i l k}^{(213)}$ instead of $\beta_{i l k}^{(123)}$ :

$$
\beta_{i j k}^{\left[\mathrm{D} 1^{\prime}\right]}\left(\omega_{1}, \omega_{2}\right)=-\sum_{h, l} W_{l h} \alpha_{h j}^{\mathrm{sub}}\left(\omega_{1}\right) \cdot \beta_{i l k}^{(213)}\left(\omega_{1}, \omega_{2}\right),
$$

so that, from equation (14):

$$
\beta_{i j k}^{[\mathrm{D} 1]}\left(\imath \omega_{1}, \imath \omega_{2}\right)+\beta_{i j k}^{\left[\mathrm{D} 1^{\prime}\right]}\left(\omega_{1}, \omega_{2}\right)=\sum_{v} \frac{A_{v}^{i j k}\left(\omega_{1}, \omega_{2}\right)}{\omega_{2}-\omega_{v}+\imath \Gamma_{v}},
$$

with:

$$
A_{v}^{i j k}\left(\omega_{1}, \omega_{2}\right)=-\frac{1}{\hbar} \sum_{h, l} \partial_{v} \alpha_{i l}\left(\omega_{3}\right) W_{l h} \alpha_{h j}^{\mathrm{sub}}\left(\omega_{1}\right) \partial_{v} \mu_{k}
$$

This result is consistent with the previous study of the SFG response of quantum dots capped with mercaptocarboxylic acid ligands [38]. The vibration amplitude $A_{v}^{i j k}$ has indeed proved to be driven by the linear susceptibility of QDs: $\alpha_{h j}^{\mathrm{sub}}\left(\omega_{1}\right)=\chi_{h j}^{\mathrm{QD}}\left(\omega_{1}\right) / N$, where $N$ is the surface density of QDs on the sample.

Henceforth, our diagrammatic approach demonstrates that it is possible to explicitly determine coupling coefficients $A_{v}$ and thus gives meaning to the phenomenological equation (2).

Diagrams of interest for 3rd-order molecular response. As recalled in the introduction (equation 3), sumfrequency generation may arise from a molecular thirdorder process characterized by the hyperpolarizability $\gamma\left(\omega_{1}, \omega_{2}, 0\right)$. Interestingly, such a 4 -rank response tensor can be formally derived from diagrams made of four molecular propagators. We thus count three interesting couples $\left(N_{\text {mol }}, N_{\text {sub }}\right)$ :

$$
\left\{\begin{array}{c}
N_{\mathrm{mol}}=4 \\
0 \leqslant V \leqslant 3
\end{array}\right\} \quad \Longrightarrow \quad(4,1) \quad(4,3) \quad(4,5) .
$$

According to Figure 4, we count 6 diagrams of the first type and 9 diagrams of the second one. In addition, 12 diagrams of type $(4,5)$ can be drawn (Figure 7 ). All these diagrams account for the molecular SFG process modified by a static electric field or a permanent dipole created by the substrate, as all of them involve at least one virtual boson carrying a zero frequency. Therefore, such diagrams may be employed to model electric-field-induced SFG at electrochemical or charged interfaces, as it is extensively studied in the literature [27-30]. Among these, only the six $(4,1)$ diagrams imply no energy exchange between molecules and substrate, and are therefeore the only ones considered by the literature. However, the loop diagrammatic method shows that additional processes involving a more complex interplay between molecules and substrates could be included in this third-order SFG response. Strictly speaking, those diagrams still translate into global $\beta_{i j k}$ functions (since $N_{p}=3$ ), but they necessarily lead to a factorized expression of the form:

$$
\beta_{i j k}\left(\omega_{1}, \omega_{2}\right)=\sum_{l} \gamma_{i j k l}\left(\omega_{1}, \omega_{2}, 0\right) U_{l},
$$

where $\mathbf{U}=\left(U_{i}\right)$ is a vector depending on the interaction hamiltonian $\mathcal{H}_{\text {int }}$ which drives the coupling between the two subsystems. In the case of electric-field-induced SFG, driven by equation (18), this vector is nothing but the static electric field $\mathbf{E}_{0}$.

Classification of composite diagrams. Within the framework of substrate/molecule systems, the last two 


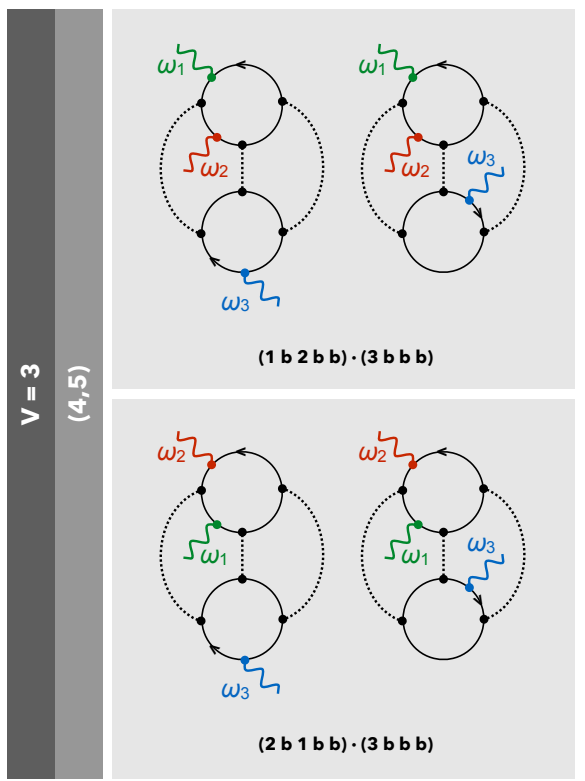

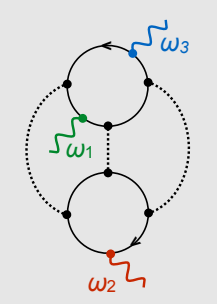

$(3 \mathrm{~b} 1 \mathrm{~b} b) \cdot(2 \mathrm{~b} b \mathrm{~b})$

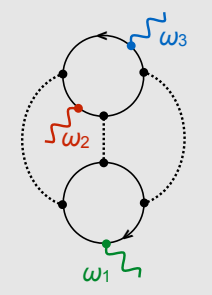

$(3 \mathrm{~b} 2 \mathrm{~b} b) \cdot(1 \mathrm{~b} b \mathrm{~b})$
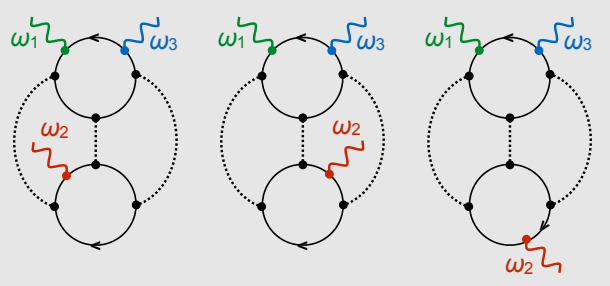

$(31 \mathrm{~b} b$ b) $\cdot(2 \mathrm{~b} b \mathrm{~b})$
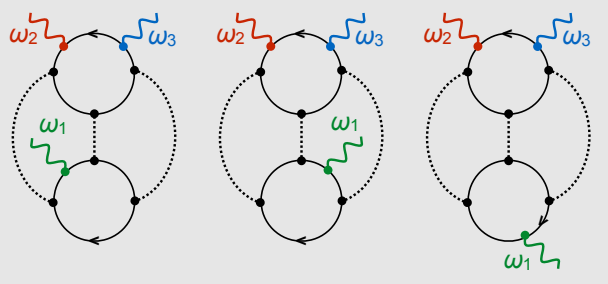

$(32 \mathrm{~b} b$ b) $\cdot(1 \mathrm{~b}$ b b )

FIG. 7. Third order molecular response. List of 2-loop diagrams of type $(4,5)$ involved in the third-order molecular response of any bipartite system. They contribute to the hyperpolarizability $\gamma_{i j k l}\left(\omega_{1}, \omega_{2}, 0\right)$ of a molecule, associated to the lower loop, under the influence of a partner (e.g. substrate, electrode, nanoparticle), associated to the upper loop.

parts above focus on the molecular response. Considering the full second-order response of the system, this is just an illustrative point of view which invites us to partition the diagrams into second-order and third-order (and so on) subsets, respectively characterized by $N_{\text {mol }}$ (or $\left.N_{\text {sub }}\right)=3$ and $N_{\text {mol }}\left(\right.$ or $\left.N_{\text {sub }}\right)=4$. In a general way, there is no limit to the orders of development when $V$ grows. If we note $Y$ the number of nodes (i.e. connected vertices) on the loop of one of the subsystems, the associated hyperpolarizability for this subsystem is then characteristic of a $(Y-1)^{\mathrm{th}}$-order process, even if it behaves like an effective second-order polarizability $\boldsymbol{\beta}$ for the total system. For instance, this is the true meaning of equation (32). Considering the decreasing magnitudes of out-of-resonance hyperpolarizabilities with their increasing order, this classification in terms of number of nodes per loop shows that the influence of most diagrams decreases when $V$ (and thus $Y$ ) grows. For the total response function, it becomes possible to discard most of the nonresonant and higher order diagrams by comparison to the main contributions.

In any case, the complete nonlinear response function of a composite system is given by the sum of all the diagrams which are physically relevant. This precisely makes sense of the phenomenological equation (3) commonly employed for describing vibrational SFG:

- the substrate contribution $\beta_{\text {sub }}$ corresponds to the sum of all the diagrams for which $N_{\text {sub }}=3$;

- the molecular contribution $\beta_{\mathrm{mol}}$ corresponds to the sum of all the diagrams for which $N_{\text {mol }}=3$;

- and the third-order contribution $\gamma$ correspond to the sum of all the diagrams for which $N_{\text {mol }}=4$.

In other words, all these terms have a common theoretical origin and can be analytically derived from the same diagrammatic method.

Extension to other processes and systems. Obviously, the loop-diagrammatic method can be enlarged to the case of systems made of more than two subunits and to all the other nonlinear optical processes. We have chosen to illustrate this formalism with SFG spectroscopy in the case of organic/inorganic systems because the understanding of the vibroelectronic coupling occurring within such systems is a controversial issue [37, 38, 5961]. Given that our approach is very fundamental and general, we expect interesting applications for the theoretical study of the second-order SHG (second harmonic generation), DFG (difference-frequency generation) and third-order Raman spectroscopies. Briefly, for a given system, the computation of SHG and DFG response functions would be very similar and mostly consist in setting $\omega_{1}=\omega_{2}$ with identical incoming photon polarizations for SHG, while adjusting energy conservation to $\omega_{3}=\omega_{1}-\omega_{2}$ and replacing annihilation by creation operator for the IR photon for DFG. Formally, the reasoning we used for the calculation of the response tensor $\boldsymbol{\beta}\left(\omega_{1}, \omega_{2}\right)$ could thus be straightforwardly applied to the other second-order optical processes. As a third-order process, Raman scattering is more complex to account for on a theoretical point of view [62]. When performed on molecules deposited on a rough metallic surface (or metallic nanoparticles), it is amplified by the surface-enhanced Raman scattering (SERS) process, whose explanation is still a matter of de- 
bate [63-66]. In this context, the present work introduces a new way of thinking for multipartite Raman processes, and SERS in particular, with the difference that the associated Feynman diagrams would consist in combining four photons to compute tensors $\gamma\left(\omega_{0},-\omega_{0}, \omega\right)$. This may seem a daunting task, but we must keep in mind that the efficiency of the diagrammatic approach lies in mixing solid-state and molecular physics with optics, through a unique formalism. For SERS, the question is precisely on the coupling between the metallic surface (or particles) and the grafted molecules. This is why we are quite confident that our method will also yield positive results for organic/inorganic systems probed by third-order optical spectroscopies.

\section{CONCLUSION}

Fundamentally, Green's functions are the proper and dedicated language of linear and nonlinear responses theories. Although they may seem tricky to use for complex systems, this difficulty can be overcome thanks to Feynman diagrams. In the case of composite systems, our method consists in building such diagrams taking into account the interactions between the different subsystems, and to apply the Feynman rules which enable to translate any diagram into the analytical expression of the associated response function.

In this article, the case of substrate/molecule interfaces probed by SFG spectroscopy was considered to illustrate the method. We enumerated in a general and exhaustive way the bipartite diagrams that it is possible to generate and gave the fundamental principles which allow their conversion into optical response functions. Practically, we explicitly shew how to establish the analytical expressions of the second-order hyperpolarizabilities for any system made of two subunits. Indeed, our results are formally independent of the precise natures of the two interacting subsystems so that they can be theoretically applied for any composite system. While the community had been failing so far to adopt a global theoretical view on the treatment of the different nonlinear optical responses within hybrid systems, here we propose a unified formalism and demonstrate its operational capability. This paves the way for concrete applications to some real bipartite systems, especially made of organic molecules in interaction with plasmonic nanoparticles, semiconductor quantum dots and charged surfaces.

\section{APPENDIX A: MATSUBARA FREQUENCIES AND RESIDUE THEOREM}

The Matsubara frequencies $\left\{\imath \omega_{\nu}\right\}_{\nu \in \mathbb{Z}}$ of fermions are defined as the poles of the Fermi-Dirac function $\rho(z)=\left(e^{\hbar b z}+1\right)^{-1}, b=1 / k_{b} T$ :

$$
\hbar \omega_{\nu}=\frac{\pi}{b}(2 \nu+1)
$$

For bosons, they are the poles of the Bose-Einstein function $\varrho_{B}(z)=\left(e^{\hbar b z}-1\right)^{-1}$ :

$$
\hbar \omega_{\gamma}=\frac{\pi}{b} 2 \gamma, \gamma \in \mathbb{Z}
$$

In this way, for bosons: $e^{\imath \hbar b \omega_{\gamma}}=1$. It means that $\rho\left(\omega \pm \imath \omega_{\gamma}\right)=\rho(\omega)$. Since photons are bosons, this identity is used to simplify the computation of optical response functions.

Besides, residue theorem allows the reduction of sums of products of imaginary time Green's functions. It states that for all meromorphic function $\phi$ characterized by $N$ simple poles $\left\{z_{u}\right\}_{1 \leqslant u \leqslant N}$ and the associated residues $\left\{r_{u}\right\}_{1 \leqslant u \leqslant N}[50,52]$ :

$$
\frac{1}{b} \sum_{\nu} \phi\left(\imath \omega_{\nu}\right)=\hbar \sum_{u} r_{u} \rho\left(z_{u}\right) .
$$

In the present article, we deal with three kinds of meromorphic functions:

$$
\begin{aligned}
\phi_{1}(z) & =\frac{1}{z-z_{1}} \\
\phi_{2}(z) & =\frac{1}{z-z_{1}} \frac{1}{z-z_{2}} \\
\phi_{3}(z) & =\frac{1}{z-z_{1}} \frac{1}{z-z_{2}} \frac{1}{z-z_{3}},
\end{aligned}
$$

wherein $z_{1}, z_{2}$ and $z_{3}$ denote the poles. The function $\phi_{1}$ is involved in one-propagator loops, $\phi_{2}$ in the computation of linear response functions, and $\phi_{3}$ in the computation of second-order response functions. From equation (35), we get:

$$
\begin{aligned}
\frac{1}{b} \sum_{\nu} \phi_{1}\left(\imath \omega_{\nu}\right) & =\hbar \rho\left(z_{1}\right) \\
\frac{1}{b} \sum_{\nu} \phi_{2}\left(\imath \omega_{\nu}\right) & =\hbar \frac{\rho\left(z_{1}\right)-\rho\left(z_{2}\right)}{z_{1}-z_{2}} \\
\frac{1}{b} \sum_{\nu} \phi_{3}\left(\imath \omega_{\nu}\right) & =\frac{\hbar \rho\left(z_{1}\right)}{\left(z_{1}-z_{2}\right)\left(z_{1}-z_{3}\right)}+\frac{\hbar \rho\left(z_{2}\right)}{\left(z_{2}-z_{1}\right)\left(z_{2}-z_{3}\right)} \\
& +\frac{\hbar \rho\left(z_{3}\right)}{\left(z_{3}-z_{1}\right)\left(z_{3}-z_{2}\right)} .
\end{aligned}
$$

These relationships are easily generalized for $\phi_{n}(z)$ with $n \geqslant 4$. 


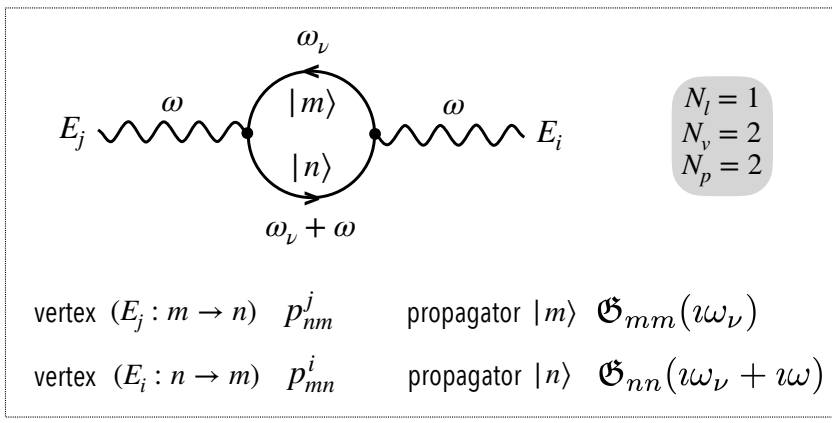

FIG. 8. Linear polarizability. Diagrammatic representation of $\alpha_{i j}(\imath \omega)$ for a simple system.

\section{APPENDIX B: DERIVATION OF THE LINEAR POLARIZABILITY}

We consider the Feynman diagram drawn in Figure 8. It is built with the vertices associated to light-matter interaction and involves an input $j$-polarized photon and an output $i$-polarized photon, both of frequency $\imath \omega$. From the Feynman rules, this diagram is converted into:

$\alpha_{i j}(\imath \omega)=\frac{(-1)^{3}}{\hbar^{2} b} \sum_{n, m ; \nu} p_{m n}^{i} p_{n m}^{j} \mathfrak{G}_{m m}\left(\imath \omega_{\nu}\right) \mathfrak{G}_{n n}\left(\imath \omega_{\nu}+\imath \omega\right)$.

Given the expressions of Green's functions:

$\alpha_{i j}(\imath \omega)=-\frac{1}{\hbar^{2}} \sum_{n, m} p_{m n}^{i} p_{n m}^{j} \frac{1}{b} \sum_{\nu} \frac{1}{\imath \omega_{\nu}-\omega_{m}} \frac{1}{\imath \omega_{\nu}-\left(\omega_{n}-\imath \omega\right)}$

Thanks to the residue theorem (Appendix A) applied for the sum over $\nu$ :

$$
\alpha_{i j}(\imath \omega)=-\frac{1}{\hbar} \sum_{n, m} p_{m n}^{i} p_{n m}^{j} \frac{\rho\left(\omega_{m}\right)-\rho\left(\omega_{n}-\imath \omega\right)}{\omega_{m}-\omega_{n}+\imath \omega} .
$$

As $\imath \omega$ is the Matsubara frequency of a photon, that is a boson, we have: $\rho\left(\omega_{n}-\imath \omega\right)=\rho\left(\omega_{n}\right)$. We eventually obtain:

$$
\alpha_{i j}(\imath \omega)=\frac{1}{\hbar} \sum_{m, n} \rho\left(\omega_{m}\right)\left(\frac{p_{n m}^{i} p_{m n}^{j}}{\imath \omega+\omega_{n m}}-\frac{p_{n m}^{j} p_{m n}^{i}}{\imath \omega-\omega_{n m}}\right) .
$$

By replacing $\imath \omega$ by $\omega+\imath 0^{+}$, we retrieve the expression of equation (11).

\section{APPENDIX C: DERIVATION OF THE MOLECULAR HYPERPOLARIZABILITY}

Here we present the method to reduce a sum over an implicit Matsubara frequency (i.e. $\omega_{\nu}$ ) from the residue theorem, applied to the SFG hyperpolarizability of a purely molecular system, and recover the essential SFG formulas of the literature expressed in the imaginary frequency space. We start from equation (13):

$$
\begin{aligned}
\beta_{i j k}^{(213)}\left(\imath \omega_{1}, \imath \omega_{2}\right)= & \frac{1}{\hbar^{3} b} \sum_{m, n, l ; \nu} p_{m n}^{i} p_{n l}^{j} p_{l m}^{k} \\
& \mathfrak{G}_{m m}\left(\imath \omega_{\nu}\right) \mathfrak{G}_{l l}\left(\imath \omega_{\nu}+\imath \omega_{2}\right) \mathfrak{G}_{n n}\left(\imath \omega_{\nu}+\imath \omega_{3}\right) .
\end{aligned}
$$

Thanks to the residue theorem (Appendix A), we reduce the sum over $\nu$ :

$$
\begin{aligned}
& \frac{1}{b} \sum_{\nu} \mathfrak{G}_{m m}\left(\imath \omega_{\nu}\right) \mathfrak{G}_{l l}\left(\imath \omega_{\nu}+\imath \omega_{2}\right) \mathfrak{G}_{n n}\left(\imath \omega_{\nu}+\imath \omega_{3}\right) \\
= & \frac{1}{b} \sum_{\nu} \frac{1}{\imath \omega_{\nu}-\omega_{m}} \frac{1}{\imath \omega_{\nu}-\left(\omega_{l}-\imath \omega_{2}\right)} \frac{1}{\imath \omega_{\nu}-\left(\omega_{n}-\imath \omega_{3}\right)} \\
= & \frac{\hbar \rho\left(\omega_{m}\right)}{\left(\omega_{m}-\omega_{l}+\imath \omega_{2}\right)\left(\omega_{m}-\omega_{n}+\imath \omega_{3}\right)} \\
& +\frac{\hbar \rho\left(\omega_{l}-\imath \omega_{2}\right)}{\left(\omega_{l}-\omega_{m}-\imath \omega_{2}\right)\left(\omega_{l}-\omega_{n}+\imath \omega_{1}\right)} \\
& +\frac{\hbar \rho\left(\omega_{n}-\imath \omega_{3}\right)}{\left(\omega_{n}-\omega_{m}-\imath \omega_{3}\right)\left(\omega_{n}-\omega_{l}-\imath \omega_{1}\right)} .
\end{aligned}
$$

Since photons are bosons: $\rho\left(\omega_{l}-\imath \omega_{2}\right)=\rho\left(\omega_{l}\right)$ and $\rho\left(\omega_{n}-\imath \omega_{3}\right)=\rho\left(\omega_{n}\right)$. We thus obtain three terms which look like those deduced from double-sided diagrams [46], with the difference that we get a term which does not resonate with $\omega_{3}$ (the second one in equation (43)). Actually, it is possible to split it in order to unveil such a resonance. Mathematically:

$$
\begin{aligned}
\frac{1}{\left(\omega_{l m}-\imath \omega_{2}\right)\left(\omega_{l n}+\imath \omega_{1}\right)} & =\frac{1}{\left(\omega_{n m}-\imath \omega_{3}\right)\left(\omega_{l n}+\imath \omega_{1}\right)} \\
& +\frac{1}{\left(\omega_{n m}-\imath \omega_{3}\right)\left(\imath \omega_{2}-\omega_{l m}\right)},
\end{aligned}
$$

with $\omega_{n m}=\omega_{n}-\omega_{m}$ and so on. As a consequence:

$$
\begin{aligned}
\beta_{i j k}^{(213)}\left(\imath \omega_{1}, \imath \omega_{2}\right)= & \\
\frac{1}{\hbar^{2}} \sum_{m, n, l} p_{m n}^{i} p_{n l}^{j} p_{l m}^{k} & {\left[\frac{\rho\left(\omega_{m}\right)}{\left(\omega_{n m}-\imath \omega_{3}\right)\left(\omega_{l m}-\imath \omega_{2}\right)}\right.} \\
& +\frac{\rho\left(\omega_{l}\right)}{\left(\omega_{n m}-\imath \omega_{3}\right)\left(\omega_{l n}+\imath \omega_{1}\right)} \\
& +\frac{\rho\left(\omega_{l}\right)}{\left(\omega_{n m}-\imath \omega_{3}\right)\left(\imath \omega_{2}-\omega_{l m}\right)} \\
& \left.+\frac{\rho\left(\omega_{n}\right)}{\left(\omega_{n m}-\imath \omega_{3}\right)\left(\omega_{n l}-\imath \omega_{1}\right)}\right] .
\end{aligned}
$$

Here, we use the labels (a1), (b2), etc. employed by R.W. Boyd [46] in his equation (3.6.18). To prove this correspondence between the loop diagram (213) and the four double-sided diagrams (a1), (b2), (a1') and (b2') we need to play with the indices, especially because in the original publication the four terms are not factorized by the dipole moments but by the density matrix coefficients $\hat{\rho}_{l l}$, formally equivalent to the Fermi-Dirac function $\rho\left(\omega_{l}\right)$, 
as explained in Appendix D. Hence, we must apply the following change of indices:

$$
\begin{aligned}
(\mathrm{a} 1) & :(m, n, l) \longrightarrow(l, n, m), \\
(\mathrm{b} 2) & :(m, n, l) \longrightarrow(n, m, l), \\
\left(\mathrm{a} 1^{\prime}\right) & :(m, n, l) \longrightarrow(n, m, l), \\
\left(\mathrm{b} 2^{\prime}\right) & :(m, n, l) \longrightarrow(m, l, n),
\end{aligned}
$$

and the formal correspondence:

$$
\rho\left(\omega_{l}\right) \longleftrightarrow \hat{\rho}_{l l}
$$

Therefore, we obtain:

$$
\begin{aligned}
& \beta_{i j k}^{(213)}\left(\imath \omega_{1}, \imath \omega_{2}\right)= \\
& \frac{1}{\hbar^{2}} \sum_{m, n, l} \hat{\rho}_{l l}\left[\frac{p_{l n}^{i} p_{n m}^{j} p_{m l}^{k}}{\left(\omega_{n l}-\imath \omega_{3}\right)\left(\omega_{m l}-\imath \omega_{2}\right)}\right. \\
&+\frac{p_{n m}^{i} p_{m l}^{j} p_{l n}^{k}}{\left(\omega_{n m}+\imath \omega_{3}\right)\left(\omega_{m l}-\imath \omega_{1}\right)} \\
&+\frac{p_{n m}^{i} p_{m l}^{j} p_{l n}^{k}}{\left(\omega_{m n}-\imath \omega_{3}\right)\left(\omega_{n l}+\imath \omega_{2}\right)} \\
&\left.+\frac{p_{m l}^{i} p_{l n}^{j} p_{n m}^{k}}{\left(\omega_{m l}+\imath \omega_{3}\right)\left(\omega_{n l}+\imath \omega_{1}\right)}\right] .
\end{aligned}
$$

To conclude, we rigorously demonstrate that a single loop diagram gathers four double-sided diagrams.

Considering the second loop diagram (123), which only consist in the permutation of the two input photons, we get $\beta_{i j k}^{(123)}$ from $\beta_{i j k}^{(213)}$ by applying the permutations:

$$
\begin{aligned}
(j, k) & \longrightarrow(k, j), \\
\left(\omega_{1}, \omega_{2}\right) & \longrightarrow\left(\omega_{2}, \omega_{1}\right),
\end{aligned}
$$

so that:

$$
\begin{aligned}
& \beta_{i j k}^{(123)}\left(\imath \omega_{1}, \imath \omega_{2}\right)= \\
& \frac{1}{\hbar^{2}} \sum_{m, n, l} \hat{\rho}_{l l}\left[\frac{p_{l n}^{i} p_{m l}^{j} p_{n m}^{k}}{\left(\omega_{n l}-\imath \omega_{3}\right)\left(\omega_{m l}-\imath \omega_{1}\right)}\right. \\
&+\frac{p_{n m}^{i} p_{l n}^{j} p_{m l}^{k}}{\left(\omega_{n m}+\imath \omega_{3}\right)\left(\omega_{m l}-\imath \omega_{2}\right)} \\
&+\frac{p_{n m}^{i} p_{l n}^{j} p_{m l}^{k}}{\left(\omega_{m n}-\imath \omega_{3}\right)\left(\omega_{n l}+\imath \omega_{1}\right)} \\
&\left.+\frac{p_{m l}^{i} p_{n m}^{j} p_{l n}^{k}}{\left(\omega_{m l}+\imath \omega_{3}\right)\left(\omega_{n l}+\imath \omega_{2}\right)}\right] .
\end{aligned}
$$

Now that we have evidenced the correspondence between loop and double-sided Feynman diagrams, we can use our formalism to derive the resonant expression of the molecular hyperpolarizability when $\omega_{2}$ is an infrared frequency. Indeed, in the case of visible-infrared vibrational SFG spectroscopy, the nonlinear response is dominated by the terms which exhibit a vibrational resonance with respect to the infrared frequency $\omega_{2}$. This corresponds to the sum of (a1) and (b1):

$$
\begin{aligned}
& \beta_{i j k}\left(\imath \omega_{1}, \imath \omega_{2}\right)= \\
& \frac{1}{\hbar^{2}} \sum_{m, n, l}\left[\frac{p_{l n}^{i} p_{n m}^{j}}{\left(\omega_{n l}-\imath \omega_{3}\right)}+\frac{p_{n m}^{i} p_{l n}^{j}}{\left(\omega_{n m}+\imath \omega_{3}\right)}\right] \frac{\hat{\rho}_{l l} p_{m l}^{k}}{\left(\omega_{m l}-\imath \omega_{2}\right)} .
\end{aligned}
$$

Assuming that the molecule is in its ground state $|0\rangle$ at equilibrium, i.e. $\hat{\rho}_{l l}=\delta_{l, 0}$ :

$$
\begin{aligned}
& \beta_{i j k}\left(\imath \omega_{1}, \imath \omega_{2}\right)= \\
& \frac{1}{\hbar^{2}} \sum_{m, n}\left[\frac{p_{0 n}^{i} p_{n m}^{j}}{\left(\omega_{n 0}-\imath \omega_{3}\right)}+\frac{p_{n m}^{i} p_{0 n}^{j}}{\left(\omega_{n m}+\imath \omega_{3}\right)}\right] \frac{p_{m 0}^{k}}{\left(\omega_{m 0}-\imath \omega_{2}\right)} .
\end{aligned}
$$

First, $\omega_{2}$ is an infrared frequency and resonates with $\omega_{m 0}$ if $m$ depicts a vibrational state $|v\rangle$ of the molecule. Second, $\omega_{3}$ is a visible frequency, which implies that $\omega_{n 0}$ and $\omega_{n v} \approx \omega_{n 0}$ are electronic transitions: the integer $n$ thus describes electronic states $|e\rangle$ :

$$
\begin{aligned}
& \beta_{i j k}\left(\imath \omega_{1}, \imath \omega_{2}\right)= \\
& \frac{1}{\hbar^{2}} \sum_{e, v}\left[\frac{p_{0 e}^{i} p_{e v}^{j}}{\left(\omega_{e 0}-\imath \omega_{3}\right)}+\frac{p_{0 e}^{j} p_{e v}^{i}}{\left(\omega_{e 0}+\imath \omega_{3}\right)}\right] \frac{p_{v 0}^{k}}{\left(\omega_{v 0}-\imath \omega_{2}\right)} .
\end{aligned}
$$

The sum over $e$ is known to give the polarizability operator [58] (Appendix E):

$$
\begin{gathered}
\frac{1}{\hbar} \sum_{e}\left[\frac{p_{0 e}^{i} p_{e v}^{j}}{\left(\omega_{e 0}-\imath \omega_{3}\right)}+\frac{p_{0 e}^{j} p_{e v}^{i}}{\left(\omega_{e 0}+\imath \omega_{3}\right)}\right] \\
=\left\langle 0\left|\hat{\alpha}_{i j}\left(\imath \omega_{3}\right)\right| v\right\rangle=\left.\sqrt{\frac{\hbar}{2 \omega_{v}}} \frac{\partial \alpha_{i j}}{\partial Q_{v}}\right|_{0} ^{\left(\omega_{3}\right)} .
\end{gathered}
$$

Moreover:

$$
p_{0 v}^{k}=\left\langle 0\left|\hat{p}^{k}\right| v\right\rangle=\left.\sqrt{\frac{\hbar}{2 \omega_{v}}} \frac{\partial p^{k}}{\partial Q_{v}}\right|_{0} .
$$

Eventually, we obtain:

$$
\beta_{i j k}\left(\omega_{1}, \omega_{2}\right)=\left.\left.\sum_{v} \frac{1}{2 \omega_{v}} \frac{\partial p^{k}}{\partial Q_{v}}\right|_{0} \frac{\partial \alpha_{i j}}{\partial Q_{v}}\right|_{0} ^{\left(\omega_{3}\right)} \frac{1}{\omega_{2}-\omega_{v}+\imath 0^{+}},
$$

which corresponds to equation (14).

\section{APPENDIX D: RELATIONSHIP BETWEEN GREEN-MATSUBARA AND DENSITY MATRIX FORMALISMS}

In conventional nonlinear optics, the quantum states $|s\rangle$ of a given system (atom, molecule, nanoparticle, solid) 
are defined as a linear combination of eigenstates $|i\rangle$ (Eq. (3.3.3) in [46]):

$$
|s\rangle=\sum_{i} a_{i, s}|i\rangle .
$$

Here we consider the eigenstates $|i\rangle$ as 1-particle states (associated to fermion annihilation/creation operators $\left.c_{i} / c_{i}^{\dagger}\right)$, so that $|s\rangle$ corresponds to a certain filling of the 1-particle states. The associated hamiltonian reads:

$$
\mathcal{H}=\sum_{i} \varepsilon_{i} c_{i}^{\dagger} c_{i},
$$

and the total energy in state $|s\rangle$ is $E_{s}=\langle s|\mathcal{H}| s\rangle$.

As individual states $|i\rangle$ are fermion states, the occupation rate $n_{i, s}=\left\langle s\left|c_{i}^{\dagger} c_{i}\right| s\right\rangle$ of each 1-particle state $|i\rangle$ in the collective state $|s\rangle$ is either zero or one. The total number of fermions of the system is then $N=\sum_{i} n_{i, s}$, and:

$$
\left|a_{i, s}\right|^{2}=\frac{n_{i, s}}{N},
$$

to satisfy the normalization condition:

$$
\langle s \mid s\rangle=\sum_{i}\left|a_{i, s}\right|^{2}=1 .
$$

The density matrix $\hat{\rho}$ of the system is henceforth defined on the eigenstates $|i\rangle$ from the probability $P(s)$ to observe the system in state $|s\rangle$, and for diagonal terms we have:

$$
\hat{\rho}_{i i}=\sum_{s} P(s)\left|a_{i, s}\right|^{2}=\frac{1}{N} \sum_{s} P(s) n_{i, s} .
$$

It corresponds to the mean occupation rate of the 1particle state $|i\rangle$ over all the collective states $|s\rangle$.

The collective states $|s\rangle$ (thus defined in the canonical ensemble) are analogous to the Fock states in solid state physics (within the grand canonical ensemble). The Fermi-Dirac distribution $\rho(\varepsilon)$ is defined by:

$$
\rho\left(\varepsilon_{i}\right)=\frac{1}{Z} \operatorname{tr}\left(e^{-b \mathcal{H}} c_{i}^{\dagger} c_{i}\right)=\frac{1}{Z} \sum_{s} e^{-b E_{s}} n_{i, s},
$$

with:

$$
Z=\operatorname{tr}\left(e^{-b \mathcal{H}}\right)=\sum_{s} e^{-b E_{s}} .
$$

At thermal equilibrium, the probability $P(s)$ is driven by the Boltzmann distribution:

$$
P(s)=\frac{e^{-b E_{s}}}{Z} .
$$

Therefore, there is a link between the density matrix $\hat{\rho}$, as defined in equation (58), and the Fermi-Dirac distribution:

$$
\hat{\rho}_{i i}=\frac{1}{N} \rho\left(\varepsilon_{i}\right)
$$

With our diagrammatic method, we compute, using the loop diagrams employed in solid-state physics, the optical response functions of $N$ fermions whose statistics is driven by $\rho(\varepsilon)$. In the atomic and molecular formalism of nonlinear optics, the double-sided diagrams lead to the computation of the optical response functions of a single entity (atom or molecule) driven by $\hat{\rho}$. This entity is equally defined as a collection of fermionic states filled with $N$ fermions. The $1 / N$ factor in Eq. (62) only reflects these different points of view. Formally, the response functions computed with loop diagrams can be translated into conventional response functions (obtained from double-sided diagrams) through the substitution of $\rho\left(\varepsilon_{i}\right)$ by $\hat{\rho}_{i i}$.

\section{APPENDIX E: ELECTRONIC POLARIZABILITY OPERATOR}

We may rewrite equation (41) in the following form, taking into account Appendix D:

$$
\begin{aligned}
\alpha_{i j}(\imath \omega) & =\sum_{m} \hat{\rho}_{m m} \frac{1}{\hbar} \sum_{n}\left(\frac{p_{n m}^{i} p_{m n}^{j}}{\imath \omega+\omega_{n m}}-\frac{p_{n m}^{j} p_{m n}^{i}}{\imath \omega-\omega_{n m}}\right) \\
& =\sum_{m} \hat{\rho}_{m m}\left\langle m\left|\frac{1}{\hbar} \sum_{n}\left(\frac{\hat{p}^{j}|n\rangle\langle n| \hat{p}^{i}}{\omega_{n m}+\imath \omega}+\frac{\hat{p}^{i}|n\rangle\langle n| \hat{p}^{j}}{\omega_{n m}-\imath \omega}\right)\right| m\right\rangle .
\end{aligned}
$$

Considering that, at thermal equilibrium, only the electronic ground state $|0\rangle$ is populated, the density matrix satisfies $\hat{\rho}_{m m}=\delta_{m 0}$ :

$$
\alpha_{i j}(\imath \omega)=\left\langle 0\left|\frac{1}{\hbar} \sum_{n}\left(\frac{\hat{p}^{j}|n\rangle\langle n| \hat{p}^{i}}{\omega_{n 0}+\imath \omega}+\frac{\hat{p}^{i}|n\rangle\langle n| \hat{p}^{j}}{\omega_{n 0}-\imath \omega}\right)\right| 0\right\rangle
$$

It is thus possible to define the equilibrium polarizability operator $\hat{\alpha}_{i j}(\imath \omega)$ as:

$$
\hat{\alpha}_{i j}(\imath \omega)=\frac{1}{\hbar} \sum_{n}\left(\frac{\hat{p}^{i}|n\rangle\langle n| \hat{p}^{j}}{\omega_{n 0}-\imath \omega}+\frac{\hat{p}^{j}|n\rangle\langle n| \hat{p}^{i}}{\omega_{n 0}+\imath \omega}\right)
$$

For a molecule described by its electronic and vibrational states, respectively depicted by $|e\rangle$ and $|v\rangle$, this operator can be reduced to the sum over the electronic states as soon as $\omega$ is an optical frequency belonging to the visible spectral range. In SFG spectroscopy, this is the case of $\omega_{3}$ (as written in Appendix C):

$$
\hat{\alpha}_{i j}\left(\imath \omega_{3}\right)=\frac{1}{\hbar} \sum_{e}\left(\frac{\hat{p}^{i}|e\rangle\langle e| \hat{p}^{j}}{\omega_{e 0}-\imath \omega_{3}}+\frac{\hat{p}^{j}|e\rangle\langle e| \hat{p}^{i}}{\omega_{e 0}+\imath \omega_{3}}\right) .
$$

In this case, $|0\rangle$ is the vibronic (electronic + vibrational) ground state, from which any optical transition starts when the system is at equilibrium. In Appendix C, equation (51) involves $\left\langle 0\left|\hat{\alpha}_{i j}\left(\imath \omega_{3}\right)\right| v\right\rangle$, that is the mean value of the electronic polarizability between the vibronic ground state of the molecule and any vibrational state $|v\rangle$ belonging to the electronic ground state. 
[1] R. J. Glauber, Nobel Lecture: 100 years of light quanta, Rev. Mod. Phys. 78, 1267 (2006).

[2] N. Bloembergen, Non-Linear Optics and Spectroscopy, Science 216, 1057 (1982).

[3] P. A. Franken and J. F. Ward, Optical harmonics and nonlinear phenomena, Rev. Mod. Phys. 37, 1 (1963).

[4] P. A. Franken, A. E. Hill, C. W. Peters, and G. Weinreich, Generation of Optical Harmonics, Phys. Rev. Lett. 7, 118 (1961).

[5] J. M. Chen, J. R. Bower, C. S. Wang, and C. H. Lee, Optical second-harmonic generation from submonolayer Na-covered Ge surfaces, Optics Communications 9, 132 (1973).

[6] P. Guyot-Sionnest, J. H. Hunt, and Y. R. Shen, Sumfrequency vibrational spectroscopy of a Langmuir film: Study of molecular orientation of a two-dimensional system, Phys. Rev. Lett. 59, 1597 (1987).

[7] Y. R. Shen, Surface properties probed by secondharmonic and sum-frequency generation, Nature 337, 519 (1989).

[8] M. Bonn, C. Hess, S. Funk, J. H. Miners, B. N. J. Persson, M. Wolf, and G. Ertl, Femtosecond surface vibrational spectroscopy of co adsorbed on $\mathrm{ru}(001)$ during desorption, Phys. Rev. Lett. 84, 4653 (2000).

[9] I. V. Stiopkin, C. Weeraman, P. A. Pieniazek, F. Y. Shalhout, J. L. Skinner, and A. V. Benderskii, Hydrogen bonding at the water surface revealed by isotopic dilution spectroscopy, Nature 474, 192 (2011).

[10] G. Glockler, The Raman Effect, Rev. Mod. Phys. 15, 111 (1943).

[11] H. Xu, E. J. Bjerneld, M. Kall, and L. Borjesson, Spectroscopy of single hemoglobin molecules by surface enhanced raman scattering, Phys. Rev. Lett. 83, 4357 (1999).

[12] K.-K. Hung, U. Stege, and D. K. Hore, IR Absorption, Raman Scattering, and IR-Vis Sum-Frequency Generation Spectroscopy as Quantitative Probes of Surface Structure, Appl. Spec. Rev. 50, 351 (2015).

[13] M. B. Raschke, M. Hayashi, S. H. Lin, and Y. R. Shen, Doubly-resonant sum-frequency generation spectroscopy for surface studies, Chem. Phys. Lett. 359, 367 (2002).

[14] L. Dreesen, C. Humbert, Y. Sartenaer, Y. Caudano, C. Volcke, A. A. Mani, A. Peremans, P. A. Thiry, S. Hanique, and J.-M. Frère, Electronic and Molecular Properties of an Adsorbed Protein Monolayer Probed by Two-Color Sum-Frequency Generation Spectroscopy, Langmuir 20, 7201 (2004).

[15] J. Guthmuller and B. Champagne, Resonance raman scattering of rhodamine $6 \mathrm{G}$ as calculated by timedependent density functional theory: Vibronic and solvent effects, J. Phys. Chem. A 112, 3215 (2008).

[16] B. Busson, M. Farhat, P.-J. Nini Teunda, S. Roy, T. Jarisz, and D. K. Hore, All-experimental analysis of doubly resonant sum-frequency generation spectra: Application to aggregated rhodamine films, J. Chem. Phys. 154, 224704 (2021).

[17] C. G. Tetsassi Feugmo, V. Liégeois, Y. Caudano, F. Cecchet, and B. Champagne, Probing alkylsilane molecular structure on amorphous silica surfaces by sum frequency generation vibrational spectroscopy: First-principles calculations, J. Chem. Phys. 150, 074703 (2019).
[18] A. Peremans, Y. Caudano, P. A. Thiry, P. Dumas, W. Q. Zhang, A. L. Rille, and A. Tadjeddine, Electronic Tuning of Dynamical Charge Transfer at an Interface: K Doping of $\mathrm{C}_{60} / \mathrm{Ag}(111)$, Phys. Rev. Lett. 78, 2999 (1997).

[19] Y. Caudano, C. Silien, C. Humbert, L. Dreesen, A. A. Mani, A. Peremans, and P. A. Thiry, Electron-phonon couplings at $\mathrm{C}_{60}$ interfaces: a case study by two-color, infrared-visible sum-frequency generation spectroscopy, J. Electron Spectroscop. Relat. Phenom. 129, 139 (2003).

[20] D. Elsenbeck, S. K. Das, and L. Velarde, Substrate influence on the interlayer electron-phonon couplings in fullerene films probed with doubly-resonant SFG spectroscopy, Phys. Chem. Chem. Phys. 19, 18519 (2017).

[21] K. C. Chou, S. Westerberg, Y. R. Shen, P. N. Ross, and G. A. Somorjai, Probing the charge-transfer state of CO on $\mathrm{Pt}(111)$ by two-dimensional infrared-visible sum frequency generation spectroscopy, Phys. Rev. B 69, 153413 (2004).

[22] L. Dalstein, A. Revel, C. Humbert, and B. Busson, Nonlinear optical response of a gold surface in the visible range: A study by two-color sum-frequency generation spectroscopy. I. Experimental determination, J. Chem. Phys. 148, 134701 (2018).

[23] L. Dalstein and B. Busson, Nonlinear optical response of a gold surface in the visible range: A study by twocolor sum-frequency generation spectroscopy. II. Model for metal nonlinear susceptibility, J. Chem. Phys. 149, 034701 (2018).

[24] L. Dalstein and B. Busson, Nonlinear optical response of a gold surface in the visible range: A study by two-color sum-frequency generation spectroscopy. III. Simulations of the experimental SFG intensities, J. Chem. Phys. 149, 154701 (2018).

[25] L. Dreesen, C. Humbert, M. Celebi, J. J. Lemaire, A. A. Mani, P. A. Thiry, and A. Peremans, Influence of the metal electronic properties on the sum-frequency generation spectra of dodecanethiol self-assembled monolayers on $\mathrm{Pt}(111), \mathrm{Ag}(111)$ and $\mathrm{Au}(111)$ single crystals, Applied Physics B 74, 621 (2002).

[26] B. Bozzini, L. D'Urzo, C. Mele, B. Busson, C. Humbert, and A. Tadjeddine, Doubly Resonant Sum Frequency Generation Spectroscopy of Adsorbates at an Electrochemical Interface, J. Phys. Chem. C 112, 11791 (2008).

[27] P. E. Ohno, S. A. Saslow, H.-F. Wang, F. M. Geiger, and K. B. Eisenthal, Phase-referenced nonlinear spectroscopy of the $\alpha$-quartz/water interface, Nat. Commun. 7, 13587 (2016).

[28] Y.-C. Wen, S. Zha, X. Liu, S. Yang, P. Guo, G. Shi, H. Fang, Y. R. Shen, and C. Tian, Unveiling Microscopic Structures of Charged Water Interfaces by SurfaceSpecific Vibrational Spectroscopy, Phys. Rev. Lett. 116, 016101 (2016).

[29] P. E. Ohno, H.-F. Wang, and F. M. Geiger, Second-order spectral lineshapes from charged interfaces, Nat. Commun. 8, 1032 (2017).

[30] S. Pezzotti, D. R. Galimberti, Y. R. Shen, and M.-P. Gaigeot, Structural definition of the BIL and DL: a new universal methodology to rationalize non-linear $\chi^{(2)}(\omega)$ SFG signals at charged interfaces, including $\chi^{(3)}(\omega)$ contributions, Phys. Chem. Chem. Phys. 20, 5190 (2018).

[31] A. N. Bordenyuk, C. Weeraman, A. Yatawara, H. D. Jayathilake, I. Stiopkin, Y. Liu, and A. V. Benderskii, Vibrational Sum Frequency Generation Spectroscopy of 
Dodecanethiol on Metal Nanoparticles, J. Phys. Chem. C 111, 8925 (2007).

[32] C. Humbert, T. Noblet, L. Dalstein, B. Busson, and G. Barbillon, Sum-Frequency Generation Spectroscopy of Plasmonic Nanomaterials: A Review, Materials 12, 836 (2019).

[33] L. Dalstein, M. B. Haddada, G. Barbillon, C. Humbert, A. Tadjeddine, S. Boujday, and B. Busson, Revealing the Interplay between Adsorbed Molecular Layers and Gold Nanoparticles by Linear and Nonlinear Optical Properties, J. Phys. Chem. C 119, 17146 (2015).

[34] D. Lis, Y. Caudano, M. Henry, S. DemoustierChampagne, E. Ferain, and F. Cecchet, Selective Plasmonic Platforms Based on Nanopillars to Enhance Vibrational Sum-Frequency Generation Spectroscopy, Adv. Optical Mater. 1, 244 (2013).

[35] L. Dalstein, C. Humbert, M. Ben Haddada, S. Boujday, G. Barbillon, and B. Busson, The Prevailing Role of Hotspots in Plasmon-Enhanced Sum-Frequency Generation Spectroscopy, J. Phys. Chem. Lett. 10, 7706 (2019).

[36] M. Linke, M. Hille, M. Lackner, L. Schumacher, S. Schlücker, and E. Hasselbrink, Plasmonic Effects of Au Nanoparticles on the Vibrational Sum Frequency Spectrum of 4-Nitrothiophenol, J. Phys. Chem. C 123, 24234 (2019).

[37] C. Humbert, A. Dahi, L. Dalstein, B. Busson, M. Lismont, P. Colson, and L. Dreesen, Linear and nonlinear optical properties of functionalized CdSe quantum dots prepared by plasma sputtering and wet chemistry, J. Coll. Interf. Sci. 445, 69 (2015).

[38] T. Noblet, L. Dreesen, S. Boujday, C. Méthivier, B. Busson, A. Tadjeddine, and C. Humbert, Semiconductor quantum dots reveal dipolar coupling from exciton to ligand vibration, Commun. Chem. 1, 76 (2018).

[39] T. Noblet, S. Boujday, C. Méthivier, M. Erard, J. Hottechamps, B. Busson, and C. Humbert, Two-Dimensional Layers of Colloidal CdTe Quantum Dots: Assembly, Optical Properties, and Vibroelectronic Coupling, J. Phys. Chem. C 124, 25873 (2020).

[40] M. Hayashi, S. H. Lin, M. B. Raschke, and Y. R. Shen, A Molecular Theory for Doubly Resonant IR-UV-vis Sum-Frequency Generation, J. Phys. Chem. A 106, 2271 (2002).

[41] B. Busson, Doubly-resonant SFG and DFG spectroscopies: an analytic model for data analysis including distorted and rotated vibronic levels. I. Theory, J. Chem. Phys. (2020).

[42] B. Busson, Doubly-resonant SFG and DFG spectroscopies: an analytic model for data analysis including distorted and rotated vibronic levels. II. Applications, J. Chem. Phys. (2020).

[43] B. Busson and L. Dalstein, Sum-frequency spectroscopy amplified by plasmonics: The small particle case, J. Phys. Chem. C 123, 26597 (2019).

[44] Y. R. Shen, Nonlinear optical studies of surfaces, Appl. Phys. A 59, 541 (1994).

[45] F. Vidal, B. Busson, and A. Tadjeddine, Probing electronic and vibrational properties at the electrochemical interface using SFG spectroscopy: Methanol electrooxidation on Pt(110), Chem. Phys. Lett. 403, 324 (2005).
[46] R. W. Boyd, Nonlinear Optics, 2nd ed. (Academic Press, 2003).

[47] Y. R. Shen, The Principles of Nonlinear Optics (WileyInterscience, 1984).

[48] J. F. Ward, Calculation of Nonlinear Optical Susceptibilities Using Diagrammatic Perturbation Theory, Rev. Mod. Phys. 37, 1 (1965).

[49] S. Doniach and E. H. Sondheimer, Green's functions for solid state physicists (W. A. Benjamin, 1974).

[50] A. M. Zagoskin, Quantum theory of many-body systems: techniques and applications (Springer, 1998).

[51] L. P. Kadanoff and G. Baym, Quantum statistical mechanics: Green's function methods in equilibrium and nonequilibrium problems (Addison-Wesley Pub. Co., 1989).

[52] G. D. Mahan, Many-particle physics, 2nd ed. (Plenum Press, 1990).

[53] W. Greiner, J. Reinhardt, and D. A. Bromley, Quantum electrodynamics (Springer, 1994).

[54] J. E. Sipe, New green-function formalism for surface optics, J. Opt. Soc. Am. B 4, 481 (1987).

[55] D. P. Craig and T. Thirunamachandran, Molecular quantum electrodynamics: an introduction to radiationmolecule interaction (Academic Press, 1984).

[56] F. Hache, Optique non linéaire (EDP Sciences, 2016).

[57] A. C. Albrecht, On the Theory of Raman Intensities, J. Chem. Phys. 34, 1476 (1961).

[58] S. H. Lin and A. A. Villaeys, Theoretical description of steady-state sum-frequency generation in molecular adsorbates, Phys. Rev. A 50, 5134 (1994).

[59] N. K. Swenson, M. A. Ratner, and E. A. Weiss, Computational Study of the Resonance Enhancement of Raman Signals of Ligands Adsorbed to CdSe Clusters through Photoexcitation of the Cluster, J. Phys. Chem. C 120, 20954 (2016).

[60] M. T. Frederick, V. A. Amin, and E. A. Weiss, Optical Properties of Strongly Coupled Quantum Dot-Ligand Systems, J. Phys. Chem. Lett. 4, 634 (2013).

[61] E. Lifshitz, Evidence in Support of Exciton to Ligand Vibrational Coupling in Colloidal Quantum Dots, J. Phys. Chem. Lett. 6, 4336 (2015).

[62] P. L. Polavarapu, Ab Initio Vibrational Raman and Raman Optical Activity Spectra, J. Chem. Phys. 94, 8106 (1990).

[63] J. Gersten and A. Nitzan, Electromagnetic theory of enhanced Raman scattering by molecules adsorbed on rough surfaces, J. Chem. Phys. 73, 3023 (1980).

[64] M. Moskovits, Surface-enhanced spectroscopy, Rev. Mod. Phys. 57, 783 (1985).

[65] A. Otto, I. Mrozek, H. Grabhorn, and W. Akemann, Surface-enhanced Raman scattering, J. Phys. Condens. Matter 4, 1143 (1992).

[66] P. L. Stiles, J. A. Dieringer, N. C. Shah, and R. P. V. Duyne, Surface-Enhanced Raman spectroscopy, Annu. Rev. Anal. Chem. 1, 601 (2008).

\section{Acknowledgements}

The authors do want to thank Dr. A. Tadjeddine (Université Paris-Saclay, CNRS, Institut de Chimie Physique, 91405, Orsay, France), and Dr F. Hache (Laboratoire d'Optique et Biosciences, CNRS-INSERM, Ecole Polytechnique, France) for fruitful scientific discussion. 